\title{
BLOCK SOR PRECONDITIONED PROJECTION METHODS FOR KRONECKER STRUCTURED MARKOVIAN REPRESENTATIONS*
}

\author{
PETER BUCHHOLZ ${ }^{\dagger}$ AND TUĞRUL DAYAR ${ }^{\ddagger}$
}

\begin{abstract}
Kronecker structured representations are used to cope with the state space explosion problem in Markovian modeling and analysis. Currently, an open research problem is that of devising strong preconditioners to be used with projection methods for the computation of the stationary vector of Markov chains (MCs) underlying such representations. This paper proposes a block successive overrelaxation (BSOR) preconditioner for hierarchical Markovian models (HMMs ${ }^{1}$ ) that are composed of multiple low-level models and a high-level model that defines the interaction among low-level models. The Kronecker structure of an HMM yields nested block partitionings in its underlying continuous-time MC which may be used in the BSOR preconditioner. The computation of the BSOR preconditioned residual in each iteration of a preconditioned projection method becomes the problem of solving multiple nonsingular linear systems whose coefficient matrices are the diagonal blocks of the chosen partitioning. The proposed BSOR preconditioner solves these systems using sparse LU or real Schur factors of diagonal blocks. The fill-in of sparse LU factorized diagonal blocks is reduced using the column approximate minimum degree (COLAMD) ordering. A set of numerical experiments is presented to show the merits of the proposed BSOR preconditioner.
\end{abstract}

Key words. Markov chains, Kronecker structured numerical techniques, block SOR, preconditioning, projection methods, real Schur factorization, COLAMD ordering

AMS subject classifications. 60J27, 15A72, 65F10, 65F50, 65B99, 15A23, 65F05, 65F 15

DOI. $10.1137 / \mathrm{S} 1064827503425882$

1. Introduction. Markovian modeling and analysis is used extensively in evaluating the performance or reliability of existing and planned communication, computer, and manufacturing systems. For example, it may be used to determine the probability of rejecting a call in a mobile communication network, the effect of increasing the number of disks in a client-server system, or the throughput of a particular station in a flow shop. Compared to simulative techniques, the attraction for Markov chains (MCs) lies in that they provide exact results up to computer precision for performance or reliability measures through numerical analysis [41]. The systems of interest are becoming increasingly complex, which makes their modeling and quantitative analysis difficult. The major problem associated with Markovian modeling and analysis is known as state space explosion, and it refers to the fact that the number of states required to represent a complex system grows exponentially with the number of components (or subsystems) in the system. A currently popular way of dealing with this problem is to employ Kronecker [45] (or tensor) structured representations.

The concept of using Kronecker operations to define large MCs underlying structured representations appears in hierarchical Markovian models (HMMs) [8, 13, 15], or in compositional Markovian models such as stochastic automata networks (SANs)

*Received by the editors April 17, 2003; accepted for publication (in revised form) April 21, 2004; published electronically March 22, 2005. Part of this work was carried out at Dresden University of Technology, where the second author was a research fellow of the Alexander von Humboldt Foundation.

http://www.siam.org/journals/sisc/26-4/42588.html

$\dagger$ Informatik IV, Universität Dortmund, D-44221 Dortmund, Germany (peter.buchholz@cs.unidortmund.de).

${ }^{\ddagger}$ Department of Computer Engineering, Bilkent University, TR-06800 Bilkent, Ankara, Turkey (tugrul@cs.bilkent.edu.tr).

${ }^{1}$ Throughout the paper, the HMM acronym stands for hierarchical Markovian models and should not be confused with the HMM that is sometimes used for hidden Markov models. 
$[33,34,35,41]$ and different classes of superposed stochastic Petri nets (SPNs) [20, 26]. In the Kronecker structured approach, the system of interest is modeled so that it is formed of smaller interacting components, and its larger underlying MC is neither generated nor stored but rather represented as a sum of Kronecker products of the smaller component matrices. In order to analyze large, structured Markovian models efficiently, various algorithms for vector-Kronecker product multiplication are devised [14, 21, 22, 33] and used as kernels in iterative solution techniques proposed for HMMs [8, 10, 12], SANs [10, 11, 14, 33, 41, 42, 43], and superposed generalized SPNs (GSPNs) [26].

Currently an open research problem is that of devising strong preconditioners $[25,38]$ to be used with projection (or Krylov subspace) methods [38] for MCs underlying Kronecker structured representations [10, 11, 41, 42]. It is known that projection methods for sparse MCs should be used with preconditioners, such as those based on incomplete LU (ILU) factorizations, to be competitive with block successive overrelaxation (BSOR) and iterative aggregation-disaggregation (IAD) [18]. However, it is not clear how to devise ILU-type preconditioners for MCs that are in the form of sums of Kronecker products.

So far, various preconditioners have been proposed for Kronecker structured representations such as those based on truncated Neumann series [41, 42], the cheap and separable preconditioner for HMMs and compositional Markovian models [10], and circulant preconditioners for a class of SANs [16]. The Kronecker product approximate preconditioner for SANs introduced recently in [28], although encouraging, is in the form of a prototype implementation.

On the other hand, results in [18] on the computation of the stationary vector of MCs show that BSOR with suitable partitionings is a very competitive solver when compared with IAD and ILU preconditioned projection methods. BSOR is developed for SANs in [43]. Therein it is shown that the Kronecker structure of the underlying continuous-time MC (CTMC) yields nested block partitionings. Recently, a more sophisticated BSOR solver was introduced for HMMs in [12]. HMMs are composed of multiple low-level models (LLMs) and a high-level model (HLM) that defines the interaction among LLMs. As in SANs, the Kronecker structure of an HMM yields nested block partitionings in its underlying CTMC. Diagonal blocks at a particular level of the nested partitioning are all square but can have different orders in different HLM states. Consequently off-diagonal blocks that correspond to a pair of different HLM states need not be square. This is different from SANs in which all (diagonal and off-diagonal) blocks at each level of nested partitioning associated with the Kronecker structure are square and have the same order. SANs in the absence of functional transition rates are HMMs having one HLM state. Furthermore, by introducing new transitions, it is possible to transform SANs that have functional transitions to SANs without functional transitions [35]. Therefore, HMMs discussed in this paper have considerable expressive power.

The particular BSOR solver for HMMs is three-level as opposed to the usual twolevel solvers [30], since in addition to the outer BSOR iteration at the first level there exists an intermediate block Gauss-Seidel (BGS) iteration at the second level which solves the diagonal blocks of the BSOR partitioning using smaller nested diagonal blocks. But more importantly, in each HLM state the solver takes advantage of diagonal blocks with identical off-diagonal parts and diagonals differing from each other by a multiple of the identity matrix. Such diagonal blocks are referred to as candidate blocks [12] and can all utilize the same real Schur factorization [40]. Furthermore, 
when the candidate blocks satisfy some easily verified conditions, they are likely to possess sparse real Schur factors that can be constructed from the component matrices and their real Schur factors. This implies significant savings in storage during the solution process and in time during the factorization. We remark that there are many HMMs which satisfy these conditions. Furthermore, the BSOR solver utilizes the column approximate minimum degree (COLAMD) ordering [17] to reduce the fill-in of sparse LU factorized diagonal blocks.

BSOR as a preconditioner for projection methods on sparse MC problems has been considered before in $[32,18]$. Since BSOR is a preconditioned power iteration in which the preconditioning matrix (or preconditioner) is based on the block triangular part of the coefficient matrix [25], it can also be used as a preconditioner with projection methods for Kronecker structured representations. To the best of our knowledge, this is the first paper in which BSOR is considered as a preconditioner for projection methods on Kronecker structured Markovian representations. The BSOR preconditioner proposed in this paper is based on the particular implementation of BSOR in [12]. However, noticing that diagonal blocks of the BSOR partitioning need to be solved with high accuracy when BSOR is used as a preconditioner with projection methods, we present its two-level version in which the diagonal blocks are solved directly.

The next section introduces the structured description of CTMCs using HMMs in an example. The third section presents the BSOR preconditioner and discusses how the preconditioner solve at each iteration of projection methods is performed in HMMs. The fourth section explains how the diagonal blocks of the BSOR preconditioner are factorized. The fifth section describes the test problems used, which are from the areas of queueing networks, telecommunications, and (repairable) manufacturing systems. The sixth section presents the results of numerical experiments. The seventh section summarizes the results, and the eighth section concludes the paper.

2. Hierarchical Markovian models. A formal definition of HMMs can be found in [10, pp. 387-390]. Since the formal HMM notation is rather complicated and difficult to follow, we introduce HMMs in an example. Hereafter, we refer to the CTMC underlying an HMM as the matrix $Q$. This singular matrix has nonnegative off-diagonal elements and diagonal elements that are negated row sums of its offdiagonal elements.

Example 1. We consider a model of token-based scheduling in a queueing network [2] and name it qh_realcontrol. Its HLM of 9 states describes the interaction among three LLMs. LLM 1 has 203 states, LLM 2 has 164 states, and LLM 3 has 151 states. All states are numbered starting from 0 . We name the states of the HLM macrostates and those of $Q$ microstates. The mapping between LLM states and HLM states is given in Table 1. Note that macrostates in an HLM may have different numbers of microstates when LLMs have partitioned state spaces, as in this example. The microstates corresponding to each macrostate result from the crossproduct of the state space partitions of LLMs that are mapped to that particular macrostate without unreachable states. Hence, we have the number of microstates in the last column of Table 1 as the product of the cardinalities of the corresponding LLM partitions.

Seven transitions denoted by $t_{0}, t_{17}, t_{18}, t_{19}, t_{21}, t_{24}$, and $t_{27}$ take place in the HLM and affect the LLMs. The last six of these transitions are captured by the 
TABLE 1

Mapping between LLM states and HLM states in qh_realcontrol.

\begin{tabular}{|c|c|c|c|c|c|c|c|c|}
\hline HLM & LLM 1 & LLM 2 & LLM 3 & \multicolumn{5}{|c|}{ \# of microstates } \\
\hline 0 & $171: 202$ & $138: 163$ & $0: 56$ & 32 & 26 & . 57 & $=$ & 47,424 \\
\hline 1 & $0: 76$ & $138: 163$ & $127: 150$ & 77 & 26 & 24 & $=$ & 48,048 \\
\hline 2 & $77: 123$ & 100:137 & $127: 150$ & 47 & 38 & 24 & $=$ & 42,864 \\
\hline 3 & $77: 123$ & $138: 163$ & $92: 126$ & 47 & 26 & 35 & $=$ & 42,770 \\
\hline 4 & 124:170 & $62: 99$ & $127: 150$ & 47 & 38 & 24 & $=$ & 42,864 \\
\hline 5 & 124:170 & $138: 163$ & $57: 91$ & 47 & 26 & 35 & $=$ & 42,770 \\
\hline 6 & 171:202 & $0: 61$ & $127: 150$ & 32 & 62 & 24 & $=$ & 47,616 \\
\hline 7 & 171:202 & $62: 99$ & $92: 126$ & 32 & 38 & 35 & $=$ & 42,560 \\
\hline 8 & 171:202 & 100:137 & $57: 91$ & 32 & 38 & 35 & $=$ & 42,560 \\
\hline
\end{tabular}

following $(9 \times 9)$ HLM matrix:

\begin{tabular}{|c|c|c|c|c|c|c|c|c|c|}
\hline & 0 & 1 & 2 & 3 & 4 & 5 & 6 & 7 & 8 \\
\hline 0 & & & & $t_{24}$ & & $t_{19}$ & & & \\
\hline 1 & & & $t_{18}$ & & $t_{21}$ & & & & \\
\hline 2 & & & & $t_{17}$ & & & $t_{21}$ & & \\
\hline 3 & & $t_{19}$ & & & & & & $t_{21}$ & \\
\hline 4 & & & & & & $t_{27}$ & $t_{18}$ & & \\
\hline 5 & & $t_{24}$ & & & & & & & $t_{18}$ \\
\hline 6 & & & & & & & & $t_{17}$ & $t_{27}$ \\
\hline 7 & $t_{27}$ & & & & $t_{19}$ & & & & \\
\hline 8 & $t_{17}$ & & $t_{24}$ & & & & & & \\
\hline
\end{tabular}

To each transition in the HLM matrix corresponds a Kronecker product of three (i.e., number of LLMs) LLM matrices. The matrices associated with those LLMs that do not participate in a transition are all identity. LLM 1 participates in $t_{18}, t_{19}, t_{21}$, and $t_{24}$, respectively, with the matrices $Q_{t_{18}}^{(1)}, Q_{t_{19}}^{(1)}, Q_{t_{21}}^{(1)}$, and $Q_{t_{24}}^{(1)}$; LLM 2 participates in $t_{17}, t_{18}, t_{21}$, and $t_{27}$, respectively, with the matrices $Q_{t_{17}}^{(2)}, Q_{t_{18}}^{(2)}, Q_{t_{21}}^{(2)}$, and $Q_{t_{27}}^{(2)}$; and LLM 3 participates in $t_{17}, t_{19}, t_{24}$, and $t_{27}$, respectively, with the matrices $Q_{t_{17}}^{(3)}, Q_{t_{19}}^{(3)}$, $Q_{t_{24}}^{(3)}$, and $Q_{t_{27}}^{(3)}$. In general, these matrices are very sparse and therefore held in row sparse format [41]. In this example, each of the transitions $t_{17}, t_{18}, t_{19}, t_{21}, t_{24}, t_{27}$ affects exactly two LLMs. For instance, the Kronecker product associated with $t_{24}$ in element $(0,3)$ of the HLM matrix in $(2.1)$ is

$$
Q_{t_{24}}^{(1)}(171: 202,77: 123) \otimes I_{26} \otimes Q_{t_{24}}^{(3)}(0: 56,92: 126),
$$

where $Q_{t_{24}}^{(1)}(171: 202,77: 123)$ denotes the submatrix of $Q_{t_{24}}^{(1)}$ that lies between states 171 through 202 rowwise and states 77 through 123 columnwise, $I_{26}$ denotes the identity matrix of order $26, Q_{t_{24}}^{(3)}(0: 56,92: 126)$ denotes the submatrix of $Q_{t_{24}}^{(3)}$ that lies between states 0 through 56 rowwise and states 92 through 126 columnwise, and $\otimes$ is the Kronecker product operator [45]. Hence, this particular Kronecker product yields a $(47,424 \times 42,770)$ matrix. The rates associated with the 18 transitions in (2.1) are all 10,000. The transition rates are scalars that multiply the corresponding Kronecker products.

Other than Kronecker products due to the transitions in (2.1), there is a Kronecker sum implicitly associated with each diagonal element of the HLM matrix. Each Kronecker sum is formed of three LLM matrices corresponding to local transition $t_{0}$. 
For instance, the Kronecker sum associated with element $(4,4)$ of the HLM matrix is

$$
Q_{t_{0}}^{(1)}(124: 170,124: 170) \oplus Q_{t_{0}}^{(2)}(62: 99,62: 99) \oplus Q_{t_{0}}^{(3)}(127: 150,127: 150),
$$

where $\oplus$ is the Kronecker sum operator. Each Kronecker sum is a sum of three Kronecker products in which all but one of the matrices are identity. The nonidentity matrix in each Kronecker product appears in the same position as in the Kronecker sum. That state changes do not take place in any but one of the LLM matrices with $t_{0}$ in each such Kronecker product is the reason behind naming $t_{0}$ a local transition. The particular Kronecker sum associated with element $(4,4)$ of the HLM matrix is $(42,864 \times 42,864)$.

In the HLM matrix of qh_realcontrol in (2.1), there does not exist any nonlocal transition along the diagonal. In general, this need not be so. Therefore, we introduce the following definition.

DeFINITION 2.1. In a given $H M M$, let $K$ be the number of $L L M s$, let $\mathcal{S}_{j}^{(k)}$ be the subset of states of LLM $k$ mapped to macrostate $j$, let $\mathcal{T}_{i, j}$ be the set of LLM (when $i=j$, nonlocal) transitions in element $(i, j)$ of the HLM matrix, let rate $t_{e}(i, j)$ be the rate associated with transition $t_{e} \in \mathcal{T}_{i, j}$, and let $D_{j}$ be the diagonal (correction) matrix that sums the rows of $Q$ corresponding to macrostate $j$ to zero. Then the diagonal block $(j, j)$ of $Q$ corresponding to element $(j, j)$ of the HLM matrix is given by

$$
Q_{j, j}=\bigoplus_{k=1}^{K} Q_{t_{0}}^{(k)}\left(\mathcal{S}_{j}^{(k)}, \mathcal{S}_{j}^{(k)}\right)+\sum_{t_{e} \in \mathcal{T}_{j, j}} \text { rate }_{t_{e}}(j, j) \bigotimes_{k=1}^{K} Q_{t_{e}}^{(k)}\left(\mathcal{S}_{j}^{(k)}, \mathcal{S}_{j}^{(k)}\right)+D_{j}
$$

and, when there are multiple macrostates, the off-diagonal block $(i, j)$ of $Q$ corresponding to element $(i, j)$ of the HLM matrix is given by

$$
Q_{i, j}=\sum_{t_{e} \in \mathcal{T}_{i, j}} \text { rate }_{t_{e}}(i, j) \bigotimes_{k=1}^{K} Q_{t_{e}}^{(k)}\left(\mathcal{S}_{i}^{(k)}, \mathcal{S}_{j}^{(k)}\right)
$$

When there are multiple macrostates, $Q$ is a block matrix having as many blocks in each dimension as the number of macrostates (i.e., order of the HLM matrix). The diagonal and off-diagonal blocks of this partitioning are, respectively, the $Q_{j, j}$ and $Q_{i, j}$ matrices defined by (2.2) and (2.3). The diagonal of $Q$ is formed of its negated off-diagonal row sums and may be stored explicitly or can be generated as needed. In Example 1, the second term in (2.2) is missing. Although $Q$ in qh_realcontrol is of order 399,476 and has 1,871,004 nonzeros, the Kronecker representation associated with the HMM needs to store 1 HLM matrix having 18 nonzeros and 15 LLM matrices (since identity matrices are not stored) having a total of 1,486 nonzeros.

In Table 2, we provide three nested partitionings along the diagonal of $Q$ defined by the Kronecker structure of the HMM in qh_realcontrol. The columns blks and ordr list, respectively, the number and order of blocks in each macrostate for the partitionings. Since the HLM has multiple macrostates, there exists a partitioning at level 0 . The diagonal blocks at level 0 can be partitioned further as defined by LLM 1 at level 1 (i.e., one block is defined for each state of LLM 1) and LLM 2 defines the next level of partitioning (i.e., one block is defined for each pair of states of LLM 1 and LLM 2).

The next section introduces the BSOR preconditioner for Kronecker structured representations. 
TABLE 2

Three nested partitionings along the diagonal in qh_realcontrol.

\begin{tabular}{|c|c|c|c|c|c|c|}
\hline \multirow{2}{*}{$\begin{array}{l}\text { HLM } \\
\text { state }\end{array}$} & \multicolumn{2}{|c|}{ Level 0} & \multicolumn{2}{|c|}{ Level 1} & \multicolumn{2}{|c|}{ Level 2} \\
\hline & blks & ordr & blks & ordr & blks & ordr \\
\hline 0 & 1 & 47,424 & 32 & 1,482 & 832 & 57 \\
\hline 1 & 1 & 48,048 & 77 & 624 & 2,002 & 24 \\
\hline 2 & 1 & 42,864 & 47 & 912 & 1,786 & 24 \\
\hline 3 & 1 & 42,770 & 47 & 910 & 1,222 & 35 \\
\hline 4 & 1 & 42,864 & 47 & 912 & 1,786 & 24 \\
\hline 5 & 1 & 42,770 & 47 & 910 & 1,222 & 35 \\
\hline 6 & 1 & 47,616 & 32 & 1,488 & 1,984 & 24 \\
\hline 7 & 1 & 42,560 & 32 & 1,330 & 1,216 & 35 \\
\hline 8 & 1 & 42,560 & 32 & 1,330 & 1,216 & 35 \\
\hline$\Sigma=$ & 9 & & 393 & & 13,266 & \\
\hline
\end{tabular}

3. BSOR as a preconditioner for HMMs. Our aim is to solve the singular linear system $\pi Q=0$ subject to the normalization condition $\|\pi\|_{1}=1$, where $\pi$ is the (row) stationary probability vector of $Q$. We assume that $Q$ is irreducible; hence, the stationary vector of $Q$ is also its steady state vector.

Projection methods (or Krylov subspace methods) [38] are state-of-the-art iterative solvers developed mostly in the last two decades that may also be used to solve for the stationary vector of MCs [6, 18, 23, 32, 36, 41, 37]. A concise discussion on popular projection methods and the motivation behind preconditioning may be found in [3]. A recent survey of preconditioning techniques for large sparse linear systems appears in [5]. The objective in preconditioning is to transform the linear system so that it becomes easier to solve with the iterative method at hand. To provide effective solvers, projection methods are used with preconditioners. This requires the preconditioning matrix (or preconditioner) to approximate the coefficient matrix of the original system in some sense and requires the solution of linear systems involving the preconditioner to be cheap. The need for a preconditioner becomes vital when the problem of interest is especially difficult to solve. Various types of preconditioners have been and are still being developed. Their efficiency is highly dependent on the system to be solved, and it is quite difficult to forecast which preconditioner is the best for a given system.

Results with preconditioned projection methods on MCs underlying Kronecker structured representations are reported in a number of papers [10, 11, 16, 28, 42]. The preconditioner based on truncated Neumann series [41, 42] is too computationally expensive to be effective and therefore impractical, whereas the cheap and separable preconditioner $[10,11]$ that forms (the inverse of) the preconditioner using the LLM nonlocal transition submatrices and the inverses of LLM local transition submatrices is not consistently effective. The circulant preconditioner in [16] can be used only with a certain class of SANs.

The Kronecker product approximate preconditioner for SANs introduced recently in [28], although encouraging, is in the form of a prototype implementation. In [27, pp. 100-113], numerical results with this preconditioner are presented using Matlab for nine problems, all of which are feed-forward queueing networks; two of the larger problems consider models having independent subsystems, each of which can be analyzed separately, in isolation. Yet, all test problems can be thought of as being HMMs with one macrostate and having $K$ LLMs (see Definition 2.1), a total of $E$ nonlocal transitions, and specific nonzero structure in the LLM matrices. Assuming that $T=K+2 E$, the proposed preconditioner [27, pp. 99-100] requires the computation of $K T(T+1) / 2$ 
traces of the products of pairs of LLM matrices; the solution of a nonlinear minimization problem of $K T$ variables; the computation of $K$ smaller matrices, each of which is a weighted sum of $T$ LLM matrices; and the inversion of the $K$ smaller matrices that are computed. The Kronecker product of the inverted smaller matrices forms (the inverse of) the proposed preconditioner for SANs.

Results in [27] indicate that in terms of reducing the number of iterations to convergence of projection methods, such as generalized minimal residual (GMRES) [39] and biconjugate gradient stabilized (BiCGSTAB) [44], the Kronecker product approximate preconditioner demonstrates behavior similar to that of the cheap and separable preconditioner in [10]. The difference between the two preconditioners in the number of iterations with GMRES and BiCGSTAB is not more than a few iterations in any of the nine test problems. Furthermore, there are cases in which the cheap and separable preconditioner yields fewer iterations. We also remark that in general the inverted smaller matrices in the proposed preconditioner are likely to be less sparse than the inverted LLM local transition matrices in the cheap and separable preconditioner since each inverted smaller matrix is a weighted sum of $T$ matrices, one of which is an LLM local transition matrix. Still, there seems to be some timing advantages that may be gained with the Kronecker product approximate preconditioner since the preconditioning step at each iteration with it involves a single vector-Kronecker product multiplication, whereas with the cheap and separable preconditioner it involves two multiplications. The first multiplication is with a Kronecker product having the inverses of the LLM local transition matrices as factors (which are likely to be sparser than their counterparts in the Kronecker product approximate preconditioner), and the second multiplication is with a sum of Kronecker products due to LLM nonlocal transition matrices, each of which is almost always sparse. The excess setup time of the proposed Kronecker product approximate preconditioner over the cheap and separable preconditioner is dictated by the time to solve the nonlinear minimization problem of $K T$ variables. In conclusion, it is not evident what results the Kronecker product approximate preconditioner will yield on a full-fledged implementation in sparse storage suitable for larger and more complex models.

The successive overrelaxation (SOR) method and its block version, BSOR, are preconditioned power iterations (see [41, p. 144] or [25, p. 26, pp. 147-149]) and therefore can also be used with projection methods as preconditioners. BSOR as a preconditioner for projection methods on sparse MC problems has been considered before in [18, 32]. This paper is the first in which BSOR is used as a preconditioner for projection methods on Kronecker structured Markovian representations. Note that until recently [43] it was not clear how one could implement BSOR for a sum of Kronecker products and that BSOR was introduced for HMMs very recently in [12]. Although generally inferior to incomplete LU (ILU) factorization-type preconditioners (see [36, p. 467], [32], and [18]) for sparse MCs, this study shows that the proposed BSOR implementation results in an effective preconditioner for MCs underlying large and complex Kronecker structured representations.

Since we work with row vectors, we consider a right BSOR preconditioner with relaxation parameter $w \in(0,2)$. Given a block partitioning of $Q$, let $Q$ be split in block form according to the partitioning as

$$
Q=\left(\frac{1}{w} D-U\right)-\left(\frac{1-w}{w} D+L\right)
$$

where $D,-U$, and $-L$ are square matrices, respectively, formed of the block diagonal, block strictly upper-triangular, and block strictly lower-triangular parts of $Q$. Then 
the BSOR preconditioning matrix is given by

$$
M_{B S O R}=\omega^{-1} D-U .
$$

In other words, it is the first term in (3.1) (see [25, p. 149]).

At each iteration of the underlying solver, the (row) residual vector, $r$ (which may have been computed explicitly or implicitly), is used as the right-hand side of the linear system

$$
z M_{B S O R}=r
$$

to compute the preconditioned (row) residual vector, $z$ [25, pp. 25-26].

The objective of this preconditioning step is to correct the error in the approximate solution vector at that iteration. Note that if $M_{B S O R}$ were the identity matrix, the preconditioned residual would be equal to the residual computed at that iteration. However, $M_{B S O R}$ is not the identity matrix, but rather used to obtain, hopefully, an improved solution. For instance, a partitioning that may be used with the qh_realcontrol problem in forming a BSOR preconditioner is the one having 393 diagonal blocks at level 1 (see Table 2).

ALGORITHM 1. BSOR preconditioner solve for HMMs: $z M_{B S O R}=r$.

For each macrostate $j$, sequentially:

(a) Compute negated right-hand side $b$ :

- Set $b$ by $-r_{j}$; add to $b$ product of $z_{i}$ with $Q_{i, j}$ for all $i<j$.

(b) Solve block upper-triangular part at level $l(j)$ of $Q_{j, j}$ for $z_{j}$ using $b$ as negated right-hand side:

- For each diagonal block $k$ at level $l(j)$ of $Q_{j, j}$, sequentially:

(i) Solve diagonal block $k$ at level $l(j)$ in $Q_{j, j}$ for subvector $k$ of $z_{j}$ with precomputed factors using negated subvector $k$ of $b$ as right-hand side.

(ii) If $(w \neq 1)$, set subvector $k$ of $z_{j}$ by $w$ times subvector $k$ of $z_{j}$.

(iii) Add to $b$ product of subvector $k$ of $z_{j}$ with corresponding blocks in block upper-triangular part at level $l(j)$ of $Q_{j, j}$.

Algorithm 1 is a high-level description of the preconditioner solve in (3.3) for HMMs. Note that it is possible to employ different partitioning levels in different macrostates (see the parameter $l(j)$ ). This provides considerable flexibility in choosing favorable partitionings. The (row) vectors $z_{j}$ and $r_{j}$ denote, respectively, the subvectors of $z$ and $r$ corresponding to macrostate $j$. Note that the vector $b$ needs to be as long as $z_{j}$ when macrostate $j$ is considered; hence, $b$ is allocated so that it is as long as the maximum number of microstates among all macrostates. For instance, in the $q$ h_realcontrol problem $b$ would have 48,048 elements (see Table 1). The negated right-hand side $b$ is used in Algorithm 1 since the vector-Kronecker product multiplication routine is coded so as to add onto an input vector. Therefore, right before solving a diagonal block, the appropriate subvector of $b$ is negated and used as the right-hand side. Note that if one has multiple macrostates and a level 0 partitioning, then there is only one diagonal block per macrostate, and step (b) of Algorithm 1 simplifies accordingly.

Recall Definition 2.1 in section 2 and note that Algorithm 1 solves the block uppertriangular linear system in (3.3) with coefficient matrix $M_{B S O R}$ and right-hand side $r$ for the unknown vector $z$, where $r$ and $z$ are row vectors. The block partitioning of $Q$ at level 0 is defined by the HLM matrix. Assuming that the HMM has multiple 
macrostates, each off-diagonal block $Q_{i, j}$ at level 0 is a sum of Kronecker products (see (2.3)). The number of terms in the summation is given by the cardinality of $\mathcal{T}_{i, j}$. Therefore, the update on $b$ in step (a) of Algorithm 1 due to off-diagonal block $Q_{i, j}$ above diagonal block $Q_{j, j}$ can be accomplished by multiplying $z_{i}$ with the sum of Kronecker products that form $Q_{i, j}$. This update is performed using the efficient vector-Kronecker product multiplication algorithm for each off-diagonal block $Q_{i, j}$ above $Q_{j, j}$. Step (b) in Algorithm 1 has three substeps that are performed for each diagonal block at level $l(j)$ in diagonal block $Q(j, j)$. The techniques used to perform step (b)(i) of Algorithm 1 are discussed in the next section. In passing, we remark that the linear system in step (b)(i) is solved using a direct method. Step (b)(ii) of Algorithm 1 is straightforward, and it is executed only if the relaxation parameter is different from 1.0; otherwise, it is skipped. On the other hand, the execution of step (b)(iii) is somewhat intricate and deserves explanation.

Step (b)(iii) of Algorithm 1 aids in performing the update on $b$ due to the block strictly upper-triangular part at level $l(j)$ of $Q_{j, j}$. At the $k$ th iteration of the for-loop in Algorithm 1, this update can be done in two different ways. It can be done using the off-diagonal blocks either above or to the right of diagonal block $k$ at level $l(j)$ in $Q_{j, j}$. The former approach is block column oriented, requires all subvectors of $z_{j}$ between 1 and $k$ (inclusive) to be used, and updates the $(k+1)$ st subvector of $b$. The latter approach is block row oriented, requires only subvector $k$ of $z_{j}$ to be used, but updates all subvectors of $b$ starting from $(k+1)$. The form of the update given in step (b)(iii) of Algorithm 1 is block row oriented. However, in the actual implementation, we use its block column oriented version due to ease of programming. Since each block is essentially a sum of Kronecker products, the update is realized by generating on-the-fly the multipliers for each term of the summation and that correspond to the off-diagonal blocks above diagonal block $k$ at level $l(j)$ in $Q_{j, j}$. In this way, vectorKronecker multiplications associated with zero multipliers can be skipped. We remark that at level $l(j)$ in macrostate $j$, the multipliers are determined by the corresponding element from the HLM matrix and submatrices of LLMs with indices up to and including $l(j)$, whereas the Kronecker products used in the multiplications are formed by LLM submatrices with indices $(l(j)+1)$ and larger.

Next, following section 3 in [12], we provide a summary of the implementation details of the particular BSOR preconditioner and explain how the diagonal blocks of the chosen partitioning are factorized so that they can be used in step (b)(i) of Algorithm 1.

4. BSOR preconditioner implementation. The diagonal blocks that correspond to a partitioning of an irreducible CTMC have negative diagonal elements and nonnegative off-diagonal elements. Such diagonal blocks are nonsingular [7]. Algorithm 2 in [12] describes how we set up the BSOR preconditioner for an HMM so that it can be used to accelerate the convergence of projection methods for solving the underlying CTMC. As we next explain, it may be possible to reduce the number of factorized diagonal blocks.

4.1. Benefiting from real Schur factorization. In HMMs, Kronecker sums contribute only to the diagonal of the HLM matrix. Furthermore, the contribution of a Kronecker sum associated with a macrostate is the same to all diagonal blocks in that macrostate. Therefore, under certain conditions, it is possible to have several diagonal blocks with identical off-diagonal parts and diagonals differing from each other by a multiple of the identity matrix. We name such diagonal blocks candidate 
blocks [12] in that macrostate. To detect candidate blocks, one must check conditions related to transitions that appear in the HLM matrix.

Note that the set of diagonal blocks in a macrostate may form multiple partitions of candidate blocks, where blocks in each partition satisfy the definition of candidacy, but either two blocks in different partitions have different off-diagonal parts or their diagonals do not differ from each other by a multiple of the identity matrix. Detecting all such partitions is a difficult process due to the various ways in which Kronecker products contribute to diagonal blocks. We use Algorithm 1 in [12] to detect candidate blocks in each macrostate. Although this algorithm may not detect all candidate blocks, we will be content with the ones detected since it executes rapidly and we do not want to compute more than one real Schur factorization per macrostate.

Recall that the real Schur factorization of a real nonsymmetric square matrix $B$ exists [40, p. 114] and can be written as $B=Z T Z^{T}$. The matrix $T$ is quasi-triangular, meaning it is block triangular with blocks of order 1 or 2 along the diagonal; the blocks of order 1 contain the real eigenvalues of $B$, and the blocks of order 2 contain the pairs of complex conjugate eigenvalues of $B$. On the other hand, the matrix $Z$ is orthogonal and contains the real Schur vectors of $B$. When both $T$ and $Z$ are requested, the cost of factorizing $B$ of order $m$ into real Schur form, assuming it is full, is $25 m^{3}$ [19, p. 185]. Note that $B$ can also be in the form $B=(Z P)\left(P^{T} T P\right)(Z P)^{T}$ for a permutation matrix $P$ which makes $P^{T} T P$ quasi-triangular. We assume without loss of generality that $T$ is quasi-upper-triangular.

Let $B_{1}=Z T Z^{T}$ be the real Schur factorization of the first candidate block in the macrostate under consideration. Let $B_{i}=B_{1}+\lambda_{i} I, i>1$, represent its $i$ th candidate block. Then $B_{i}=Z\left(T+\lambda_{i} I\right) Z^{T}$. Hence, all candidate blocks in the same macrostate can utilize the $T$ and $Z$ factors of the first candidate block. Consequently the solution of a nonsingular linear system whose coefficient matrix is a candidate block requires two vector-matrix multiplications and one quasi-triangular solve. All that needs to be done is to store $\lambda_{i}$ for each candidate block and the real Schur factors $T$ and $Z$ in each macrostate. When the computed real Schur factors are sparse, this implies significant storage savings compared to the LU factorization of the blocks, a reduction in the setup time of the BSOR preconditioner, and in some cases a reduction in solution time as well.

The real Schur factors of a candidate block may be obtained using the CLAPACK routine dgees $[19$, p. 185] available at [31]. This routine effectively uses two twodimensional double precision arrays, the first of which has the particular matrix on input and its $T$ factor on output, whereas the second has its $Z$ factor on output. The returned factors can be compacted and stored as sparse matrices to be used in the iterative part of a solver. However, this approach is not feasible for large candidate blocks due to time and space requirements associated with the dgees routine. The next subsection states a proposition which enables one to construct the real Schur factors from smaller submatrices so that the expensive real Schur factorization of the larger candidate blocks can be circumvented.

4.2. Candidate blocks having real eigenvalues. The following proposition in [12] specifies sufficient conditions for a candidate block to have real eigenvalues (i.e., upper-triangular $T$ factor).

Proposition 4.1. Let the real Schur factorization of the local transition submatrix of LLM $k$ in element $(j, j)$ of the HLM matrix be given by (see Definition 2.1)

$$
Q_{t_{0}}^{(k)}\left(\mathcal{S}_{j}^{(k)}, \mathcal{S}_{j}^{(k)}\right)=Z_{k} T_{k} Z_{k}^{T},
$$


where $T_{k}$ is its (quasi-)upper-triangular factor and $Z_{k}$ is its orthogonal factor. Also let $\tilde{D}_{j}$ denote the diagonal block of $D_{j}$ associated with the candidate block at level $l(j)$ in macrostate $j$. If, for macrostate $j$,

(a) each $T_{k}$ for $k>l(j)$ is upper-triangular, and

(b) each $\bigotimes_{k>l(j)}\left(Z_{k}^{T} Q_{t_{e}}^{(k)}\left(\mathcal{S}_{j}^{(k)}, \mathcal{S}_{j}^{(k)}\right) Z_{k}\right)$ that contributes to the candidate block at level $l(j)$ for all $e \in \mathcal{T}_{j, j}$ is upper-triangular, and

(c) $\left(\bigotimes_{k>l(j)} Z_{k}^{T}\right) \tilde{D}_{j}\left(\bigotimes_{k>l(j)} Z_{k}\right)$ is diagonal, then the candidate block at level $l(j)$ in macrostate $j$ has real eigenvalues.

We remark that part (a) of Proposition 4.1 is satisfied, for instance, when the LLM local transition submatrices that are mapped to macrostate $j$ for LLMs $(l(j)+1)$ and higher are triangular. Its part (b) is satisfied by all macrostates along the diagonal of the HLM matrix in many HMMs arising from closed queueing networks as in Example 1 which do not have any nonlocal transitions along the diagonal of their HLM matrices (i.e., $\mathcal{T}_{j, j}=\emptyset$ for all $j$ ). Note also that it suffices for the first nondiagonal factor in the Kronecker product of part (b) to be an upper-triangular matrix to satisfy the condition for the particular $e \in \mathcal{T}_{j, j}$ (see Appendix A in [43, pp. 181-183]). Checking part (b) of the proposition requires one to have previously computed the multipliers that multiply each Kronecker product in forming the candidate block when $l(j)>0$. However, this is something we already do in detecting candidate blocks. Finally, [12] also shows how one can check part (c) of Proposition 4.1 and build the product using orthogonal real Schur factors of LLM local transition submatrices and $\tilde{D}_{j}$.

As indicated in [12], Proposition 4.1 also suggests an approach to construct the $T$ and $Z$ factors of the candidate block that is to be real Schur factorized at level $l(j)$ in macrostate $j$ from the real Schur factors of the LLM local transition submatrices, the LLM nonlocal transition submatrices, and $D_{j}$.

4.3. Reordering LLMs. When Proposition 4.1 does not apply to the original ordering of LLMs, it may apply to a reordering of LLMs, as in the next kanban problem.

Example 2. Consider the following smaller model associated with a manufacturing system having Kanban control [29] with the submatrices

$$
\begin{gathered}
Q_{t_{0}}^{(1)}=\left(\begin{array}{ll}
0 & 1 \\
0 & 0
\end{array}\right), Q_{t_{1}}^{(1)}=\left(\begin{array}{rr}
0 & 0 \\
10 & 0
\end{array}\right), Q_{t_{2}}^{(1)}=I, Q_{t_{3}}^{(1)}=I, \\
Q_{t_{0}}^{(2)}=\left(\begin{array}{ll}
0 & 1 \\
0 & 0
\end{array}\right), Q_{t_{1}}^{(2)}=\left(\begin{array}{ll}
0 & 1 \\
0 & 0
\end{array}\right), Q_{t_{2}}^{(2)}=\left(\begin{array}{ll}
0 & 0 \\
1 & 0
\end{array}\right), Q_{t_{3}}^{(2)}=I, \\
Q_{t_{0}}^{(3)}=\left(\begin{array}{ll}
0 & 1 \\
0 & 0
\end{array}\right), Q_{t_{1}}^{(3)}=I, Q_{t_{2}}^{(3)}=\left(\begin{array}{cc}
0 & 10 \\
0 & 0
\end{array}\right), Q_{t_{3}}^{(3)}=\left(\begin{array}{rr}
0 & 0 \\
10 & 0
\end{array}\right), \\
Q_{t_{0}}^{(4)}=\left(\begin{array}{ll}
0 & 0 \\
1 & 0
\end{array}\right), Q_{t_{1}}^{(4)}=I, Q_{t_{2}}^{(4)}=I, Q_{t_{3}}^{(4)}=\left(\begin{array}{ll}
0 & 1 \\
0 & 0
\end{array}\right) .
\end{gathered}
$$

Let the corresponding HLM matrix have one state with the three transitions $t_{1}, t_{2}$, and $t_{3}$ in element $(0,0)$, and let the rates of all transitions be 1 . Then the corresponding CTMC may be obtained from

$$
Q=\bigoplus_{k=1}^{4} Q_{t_{0}}^{(k)}+\sum_{e \in\{1,2,3\}} \bigotimes_{k=1}^{4} Q_{t_{e}}^{(k)}+D
$$


where $D$ is the diagonal correction matrix that sums the rows of $Q$ to zero (see Definition 2.1) as

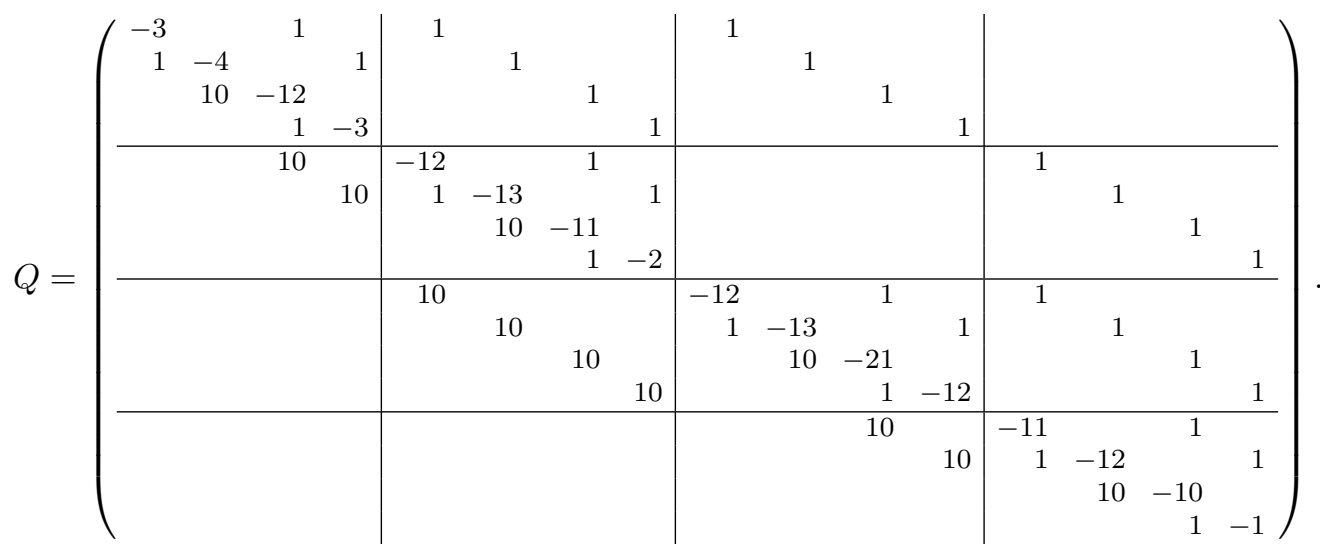

At level 2 there are 4 blocks of order 4 along the diagonal of $Q$. Observe that $Z_{3}=I_{2}$ (since $Q_{t_{0}}^{(3)}$ is strictly upper-triangular), $Q_{t_{3}}^{(3)}$ is strictly lower-triangular, and the 4 multipliers associated with the contribution of $\left(Z_{3}{ }^{T} Q_{t_{3}}^{(3)} Z_{3}\right) \otimes\left(Z_{4}{ }^{T} Q_{t_{3}}^{(4)} Z_{4}\right)$ to the 4 diagonal blocks of $Q$ at level 2 are 1 (since $Q_{t_{3}}^{(1)}=Q_{t_{3}}^{(2)}=I_{2}$ ). Hence, $Q$ does not satisfy Proposition 4.1 at level 2 since $Z_{3}^{T} Q_{t_{3}}^{(3)} Z_{3}$ is strictly lower-triangular and this contradicts part (b).

Now consider the version of the kanban model in which LLMs are reordered as (3 $\left.4 \begin{array}{lll}3 & 1\end{array}\right)$. This results in the symmetric permutation of $Q$ given by

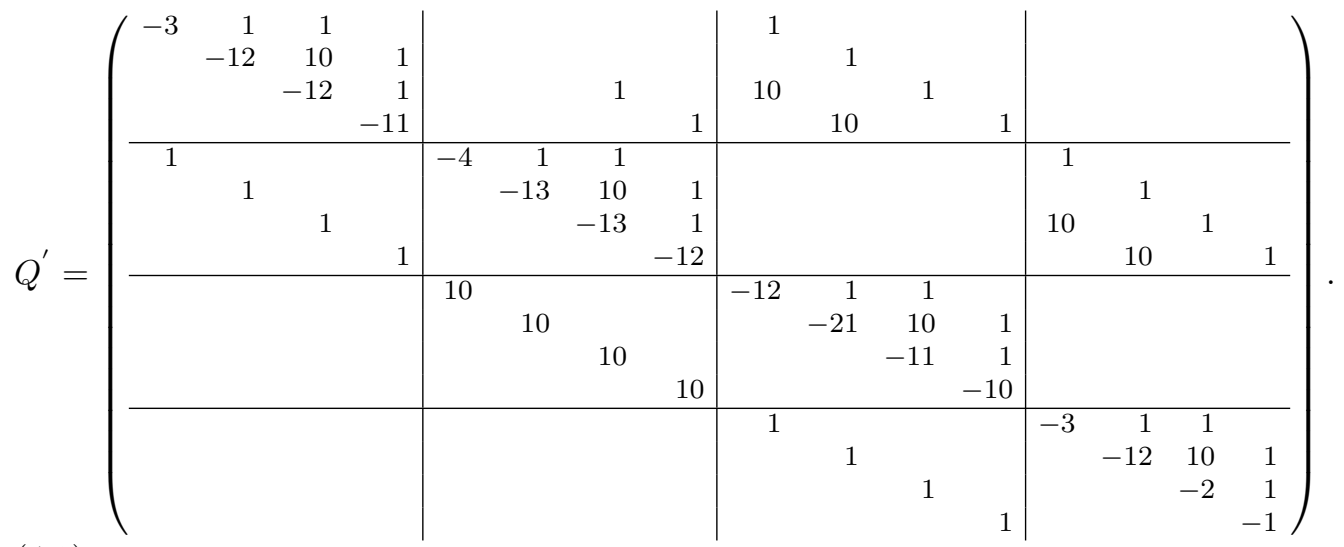

When LLMs are reordered as (3 421 ), transitions $t_{1}$ and $t_{3}$ do not pose any problems for Proposition 4.1. Regarding the 4 multipliers associated with the contribution of $\left(Z_{2}{ }^{T} Q_{t_{2}}^{(2)} Z_{2}\right) \otimes\left(Z_{1}{ }^{T} Q_{t_{2}}^{(1)} Z_{1}\right)$ to the 4 diagonal blocks of $Q^{\prime}$ that are of order 4 , they are all 0 (since both diagonal elements of $Q_{t_{2}}^{(3)}$ are 0 ). In fact, all $(4 \times 4)$ diagonal blocks of $Q^{\prime}$ are upper-triangular. Hence, the reordered CTMC satisfies Proposition 4.1 for diagonal blocks of order 4 .

As Example 2 shows, reordering LLMs may help in satisfying Proposition 4.1. It is our experience that there is considerable sparsity and structure in HMMs which result in Proposition 4.1 being satisfied in many cases (with sparse real Schur factors for 
candidate blocks). Since $Q$ never needs to be generated as a whole, reordering of LLMs is a cheap operation which requires mainly the renumbering and permutation of some components in the data structure and the checking of conditions in Proposition 4.1. Consequently several orderings may be tested to find a good ordering for the space efficient generation of the preconditioner.

4.4. When all else fails. We use the COLAMD ordering [17] on those diagonal blocks that are not candidates or that do not satisfy Proposition 4.1 to reduce the fillin produced by their sparse LU factorizations. With sparse LU factors, the solution for each nonsingular linear system in step (b)(i) of Algorithm 1 in section 3 is obtained by performing two triangular solves. See subsection 3.4 in [12] for more information on how we use COLAMD.

While experimenting, we noticed a peculiar behavior of the COLAMD ordering algorithm. When the input matrix to COLAMD is lower-triangular (for instance, as in the transpose of the diagonal blocks of $Q^{\prime}$ in (4.2)), the resulting permutation can be nonidentity. Since we use the ordering generated by COLAMD to symmetrically permute the input matrix, this implies that COLAMD destroys the ideal triangular nonzero structure of the input matrix for LU factorization, and therefore yields larger fill-in. Unfortunately, we cannot blame COLAMD for this since it does not know that we are going to perform a symmetric permutation with the column ordering it generates. Hence, we recommend not using COLAMD when the input matrix is lower-triangular and a symmetric permutation will be performed with the COLAMD ordering.

In the next section we provide the test problems used in the numerical experiments.

5. Test problems. We experiment with eight problems. The characteristics of these problems are given in Table 3. For each problem, we provide the macrostates (HLM states), the number of nonzeros in the HLM matrix $\left(n z_{H L M}\right)$ and their values (rates), the state space partition of each LLM (LLM states), the number of LLM matrices (LLM matrices), the total number of nonzeros in the LLM matrices $\left(n z_{L L M s}\right)$, the transitions in the off-diagonal part $(\mathcal{T}(i, j), i \neq j)$ and the diagonal part $(\mathcal{T}(j, j))$ of the HLM matrix, the number of states $(n)$, and the number of nonzeros $(n z)$ of the underlying CTMC.

Originally each problem was modeled as a GSPN. The GSPN model corresponding to a problem is transformed to an HMM description as discussed in $[9,13]$. The matrices for each model can be automatically generated from the GSPN specification enhanced by a partition of places to define LLMs using the APNN-toolbox [2]. The complete description to set up the Kronecker representation of $Q$ for an HMM with $K$ LLMs (see Definition 2.1) is stored in $(K+2)$ files, one for the HLM and $K$ for the LLMs. Each file contains complete information about one component, HLM or LLM, including the nonzero elements of all matrices and, for LLMs, the partition of the state space. An additional file is necessary to describe the mapping between each HLM state and the subsets of LLM states. The overall size of the files is proportional to state space sizes of components and the number of nonzero elements in the matrices of the HLM and the LLMs. Usually these numbers are negligible compared to the size of the reachable state space of the complete model which can be seen by comparing $n z_{L L M s}$ and $n$ in Table 3 . At run-time, these files are read and processed, and their contents are stored in a two-level treelike data structure. At the higher level of this structure, one has the HLM matrix. Each nonzero element of the HLM matrix forms a linked list of transitions. Each node of this linked list points to a linked list of LLM 
TABLE 3

Problems.

\begin{tabular}{|c|c|c|c|c|c|c|}
\hline Attribute & \multicolumn{3}{|c|}{ qh_realcontrol } & \multicolumn{2}{|c|}{ msmq_medium } & msmq_large \\
\hline HLM states & \multicolumn{3}{|c|}{$0: 8$} & \multicolumn{2}{|c|}{$0: 14$} & $\{0: 34\}$ \\
\hline \multirow{2}{*}{$\begin{array}{l}n z_{H L M} \\
\text { LLM } 1 \text { states }\end{array}$} & \multicolumn{3}{|c|}{$18($ rates $\in\{10,000\})$} & \multicolumn{2}{|c|}{$25($ rates $\in\{1\})$} & $75($ rates $\in\{10\})$ \\
\hline & \multicolumn{3}{|c|}{$0: 76,77: 123,124: 170,171: 202$} & \multicolumn{2}{|c|}{$0: 5,6: 16,17: 31$} & $\{0: 6,7: 19,20: 37,38: 59\}$ \\
\hline LLM 2 states & \multicolumn{3}{|c|}{$0: 61,62: 99,100: 137,138: 163$} & \multicolumn{2}{|c|}{$0: 5,6: 16,17: 31$} & $\{0: 6,7: 19,20: 37,38: 59\}$ \\
\hline LLM 3 states & \multicolumn{3}{|c|}{$0: 56,57: 91,92: 126,127: 150$} & \multicolumn{2}{|c|}{$0: 5,6: 16,17: 31$} & $\{0: 6,7: 19,20: 37,38: 59\}$ \\
\hline LLM 4 states & \multicolumn{3}{|c|}{ None } & \multicolumn{2}{|c|}{$0: 5,6: 16,17: 31$} & $\{0: 6,7: 19,20: 37,38: 59\}$ \\
\hline \multirow{2}{*}{$\begin{array}{l}\text { LLM } 5 \text { states } \\
\text { LLM matrices }\end{array}$} & \multicolumn{3}{|c|}{ None } & \multicolumn{2}{|c|}{$0: 5,6: 16,17: 31$} & $\{0: 6,7: 19,20: 37,38: 59\}$ \\
\hline & \multicolumn{3}{|c|}{15} & \multicolumn{2}{|c|}{15} & 15 \\
\hline$n z_{L L M s}$ & \multicolumn{3}{|c|}{1,486} & & & 790 \\
\hline $\mathcal{T}(i, j), i \neq j$ & $\left\{t_{1}\right.$ & $\left.t_{18}, t_{19}, t_{21}, t_{24}, t_{27}\right\}$ & & $\left\{t_{1}, t_{2}\right.$, & $\left.t_{4}, t_{5}\right\}$ & $\left\{t_{14}, t_{15}, t_{16}, t_{17}, t_{18}\right\}$ \\
\hline $\mathcal{T}(j, j)$ & Nor & & & None & & None \\
\hline$n$ & 399 & & & 358,560 & & $2,945,880$ \\
\hline$n z$ & 1,8 &, 004 & & $2,135,1$ & & $19,894,875$ \\
\hline Attribute & & kanban_medium & kanban. & large & kanban_f & \\
\hline HLM stat & & $\{0\}$ & $\{0\}$ & & $\{0: 7\}$ & \\
\hline$n z_{H L M}$ & & $3($ rates $\in\{1\})$ & 3 (rates & $\in\{1\})$ & 40 (rates & $E\{0.00001,0.0001,10\})$ \\
\hline LLM 1 ste & & $\{0: 10\}$ & $\{0: 19\}$ & & $\{0: 35,36$ & $: 80\}$ \\
\hline LLM 2 ste & & $\{0: 65\}$ & $\{0: 65\}$ & & $\{0: 35,36$ & $: 80\}$ \\
\hline LLM 3 ste & & $\{0: 65\}$ & $\{0: 65\}$ & & $\{0: 35,36$ & $: 80\}$ \\
\hline LLM 4 ste & & $\{0: 10\}$ & $\{0: 19\}$ & & $\begin{array}{l}\{0: 2,3: \\
16: 19,20\end{array}$ & $\begin{array}{l}7: 10,11: 15 \\
24,25: 29,30: 35\end{array}$ \\
\hline LLM mat & ices & 10 & 10 & & 20 & \\
\hline$n z_{L L M s}$ & & 370 & 406 & & 792 & \\
\hline $\mathcal{T}(i, j), i$ & & None & None & & $\left\{t_{4}, t_{5}, t_{6}\right.$, & $\left.t_{7}, t_{12}, t_{13}\right\}$ \\
\hline $\mathcal{T}(j, j)$ & & $\left\{t_{1}, t_{2}, t_{3}\right\}$ & $\left\{t_{1}, t_{2}, t\right.$ & & $\left\{t_{10}, t_{14}\right\}$ & \\
\hline$n$ & & 527,076 & $1,742,40$ & & $2,302,911$ & \\
\hline$n z$ & & $3,001,405$ & $10,183,3$ & & $14,313,663$ & \\
\hline Attribute & & courier_medium & & couri & large & \\
\hline HLM state & & $\{0: 9\}$ & & $\{0: 1$ & & \\
\hline$n z_{H L M}$ & & $47($ rates $\in\{1\})$ & & $65(r$ & $s \in\{1,1449$ & $3,4821.6,8771.9\})$ \\
\hline LLM 1 sta & & $\{0: 14\}$ & & $\{0: 2$ & & \\
\hline LLM 2 sta & & $\begin{array}{l}\{0: 1,2,3: 5,6: 18, \\
19: 22,23: 74,75:\end{array}$ & & $\begin{array}{l}\{0,1: \\
203:\end{array}$ & $\begin{array}{l}0,141,142 \\
, 223,224:\end{array}$ & $\begin{array}{l}201,202 \\
227,228\}\end{array}$ \\
\hline LLM 3 sta & & $\{0,1,2: 5,6,7: 26$ & $28: 87\}$ & $\{0: 1$ & & \\
\hline LLM 4 sta & & $\{0: 29\}$ & & $\begin{array}{l}\{0: 3 \\
475:\end{array}$ & $\begin{array}{l}322: 326,3 \\
, 527: 529\end{array}$ & $\begin{array}{l}27: 470,471: 474 \\
30: 542,543: 544,545\}\end{array}$ \\
\hline LLM matr & ces & 14 & & 14 & & \\
\hline$n z_{L L M s}$ & & 845 & & 2,333 & & \\
\hline $\begin{array}{l}\mathcal{T}(i, j), i \neq \\
\mathcal{T}(j, j)\end{array}$ & & $\begin{array}{l}\left\{t_{0}, t_{2}, t_{3}, t_{4}\right\} \\
\left\{t_{1}, t_{5}\right\}\end{array}$ & & $\begin{array}{l}\left\{t_{0}, t_{2}\right. \\
\left\{t_{17}, t\right.\end{array}$ & $\left.t_{29}, t_{30}\right\}$ & \\
\hline$n$ & & $\begin{array}{l}\left\{\iota_{1}, \iota_{5}\right\} \\
419,400\end{array}$ & & $\begin{array}{l}\{\iota 17, t \\
1,632,\end{array}$ & & \\
\hline$n z$ & & $2,281,620$ & & 9,732 & & \\
\hline
\end{tabular}

submatrices at the lower level that are associated with the corresponding transition and are used in forming Kronecker products. The linked lists of LLM submatrices that correspond to local transitions along the diagonal of the HLM matrix are stored separately and are used in forming Kronecker sums (see the $q$ h_realcontrol example in section 2). It is these data structures on which each iterative solver operates. Most of the presented models and the used software are available or directly accessible (for further information, see [2]).

The qh_realcontrol problem was introduced in Example 1, and the smaller version of the kanban_medium and kanban_large problems was introduced in Example 2. We also consider another version of the kanban problem in which the machines can fail, and name it kanban_fail. We consider two versions of the multiserver multiqueue problem discussed in [1] and name them msmq_medium and msmq_large. Finally, we consider two problems associated with the Courier protocol in [46] named courier_medium and courier_large (see also [9]). The CTMCs underlying all problems are irreducible. See [2] for more information about these problems. 
The $q h \_r e a l c o n t r o l, m s m q \_m e d i u m, k a n b a n \_m e d i u m$, and courier_medium problems have in the order of 100,000 states, whereas the other problems have in the order of 1,000,000 states. The kanban_medium and kanban_large problems have one macrostate; the other problems have multiple macrostates. The qh_realcontrol, $m s m q \_m e d i u m$, and $m s m q \_l a r g e$ problems do not have any nonlocal transitions along the diagonal of their HLM matrices. Regarding nonlocal transitions, each LLM in qh_realcontrol participates in four transitions, whereas each of those in $m s m q \_m e d i u m$ and $m s m q \_l a r g e$ participates in two transitions. In kanban_medium and kanban_large LLM 2 and 3 each participates in two transitions, while each of the other two LLMs participates in one transition. In kanban_fail LLM 4 participates in six transitions, LLM 1 participates in four transitions, and each of the other two LLMs participates in three transitions. In courier_medium LLM 2 and 3 each participates in four transitions, while each of the other two LLMs participates in one transition. Similar to courier_medium, in courier_large LLM 2 and 4 each participates in four transitions, while each of the other two LLMs participates in one transition. Observe that the number of LLM matrices in each problem is the sum of the number of LLMs (since there is a local transition matrix that implicitly contributes to the diagonal of the HLM matrix per LLM) and the total number of nonlocal transitions in which LLMs participate. The qh_realcontrol and kanban_fail problems are especially difficult to solve due to the existence of nonzeros in their HLM and LLM matrices that have considerably different orders of magnitude.

The reordering of LLMs in a particular problem, when desired, is carried out on its HMM description that is stored across $(K+2)$ files. By using an input permutation vector of length $K$, the reordering code generates a new HMM description corresponding to the suggested ordering of LLMs. This is performed by reading all the files associated with the HMM description, then processing and writing them in the specified order with different names. Obviously, it is critical to process and write the .spa file that stores the mapping between HLM and LLM states according to the new order of LLMs. In this way, one can rapidly generate a version of the same problem in which LLMs are reordered.

In the next section we present results of experiments with the eight problems in Table 3 .

6. Numerical experiments. There are two kinds of savings one may obtain with a BSOR preconditioner for HMMs using the ideas in this paper. These are taking advantage of real Schur factorization in candidate blocks and using COLAMD ordering in noncandidate blocks. Both of these features can be turned off in the corresponding solvers. The reordering of LLMs in an HMM can change the nested block structure of the CTMC underlying an HMM, and consequently the number and order of candidate blocks; this may lead to further savings.

We implemented the BSOR preconditioner as discussed in sections 3 and 4 in $\mathrm{C}$ as part of the APNN-toolbox [4,2]. In Table 4 we specify the ordering of LLMs (Ordering) and the associated partitionings (l, blks, cdts, ordr, ac|nc, $n z_{L U}, n z_{S c h u r}$ ) used (with BSOR) in all problems introduced in section 5. The column $l$ gives the partitioning level used across all macrostates. Hence, $l(j)=l$ for all $j$ in Algorithm 1 . The columns blks and cdts give, respectively, the number of diagonal blocks and the candidates among them. The column ordr gives the minimum and maximum order of diagonal blocks. The column ac|nc indicates whether all candidates (ac) or no candidates (nc) benefit from real Schur factorization. In the latter case (i.e., nc), all diagonal blocks are LU factorized. The columns $n z_{L U}$ and $n z_{S c h u r}$ give, respectively, 
TABLE 4

Ordering of LLMs and partitionings used.

\begin{tabular}{|c|c|c|c|c|c|c|c|c|c|c|}
\hline Problem & Ordering & $l$ & blks & cdts & ordr & $\mathrm{ac} \mid \mathrm{nc}$ & \multicolumn{2}{|r|}{$n z_{L U}$} & \multicolumn{2}{|c|}{$n z_{S c h u r}$} \\
\hline \multirow{3}{*}{ qh_realcontrol } & $\left(\begin{array}{lll}1 & 2 & 3\end{array}\right)$ & 1 & 393 & 393 & $624-1,488$ & $\mathrm{nc}$ & $\mathrm{n} / \mathrm{o}$ & $3,222,355$ & 0 & $(0)$ \\
\hline & & & & & & $\mathrm{nc}$ & $\mathrm{cmd}$ & $2,166,460$ & 0 & (0) \\
\hline & & & & & & $\mathrm{ac}$ & $\mathrm{n} / \mathrm{o}$ & 0 & 50,725 & $(399,476)$ \\
\hline msmq_medium & $\left(\begin{array}{lllll}1 & 2 & 3 & 4 & 5\end{array}\right)$ & 2 & 913 & 913 & $216-726$ & $\mathrm{ac}$ & $\mathrm{n} / \mathrm{o}$ & 0 & 40,425 & $(358,560)$ \\
\hline \multirow[t]{3}{*}{ msmq_large } & $\left(\begin{array}{lllll}1 & 2 & 3 & 4 & 5\end{array}\right)$ & 2 & 3,621 & 3,621 & $343-2,197$ & nc & $\mathrm{n} / \mathrm{o}$ & $52,011,379$ & 0 & $(0)$ \\
\hline & & & & & & nc & $\mathrm{cmd}$ & $33,980,103$ & 0 & $(0)$ \\
\hline & & & & & & ac & $\mathrm{n} / \mathrm{o}$ & 0 & 214,629 & $(2,945,880)$ \\
\hline \multirow[t]{2}{*}{ kanban_medium } & $\left(\begin{array}{llll}3 & 4 & 2 & 1\end{array}\right)$ & 2 & 726 & 121 & 726 & $\mathrm{ac}$ & $\mathrm{n} / \mathrm{o}$ & $1,537,305$ & 3,267 & $(87,846)$ \\
\hline & & & & & & ac & cmd & $3,392,235$ & 3,267 & $(87,846)$ \\
\hline \multirow[t]{2}{*}{ kanban_large } & $\left(\begin{array}{llll}3 & 4 & 2 & 1\end{array}\right)$ & 2 & 1,320 & 220 & 1,320 & ac & $\mathrm{n} / \mathrm{o}$ & $5,190,900$ & 6,039 & $(290,400)$ \\
\hline & & & & & & $\mathrm{ac}$ & $\mathrm{cmd}$ & $14,140,500$ & 6,039 & $(290,400)$ \\
\hline \multirow[t]{4}{*}{ kanban_fail } & $\left(\begin{array}{llll}1 & 2 & 3 & 4\end{array}\right)$ & 2 & 13,122 & 2,349 & $135-225$ & nc & $\mathrm{n} / \mathrm{o}$ & $13,471,515$ & 0 & $(0)$ \\
\hline & & & & & & nc & $\mathrm{cmd}$ & $8,588,025$ & 0 & $(0)$ \\
\hline & & & & & & $\mathrm{ac}$ & $\mathrm{n} / \mathrm{o}$ & $11,177,577$ & 6,248 & $(431,568)$ \\
\hline & & & & & & $\mathrm{ac}$ & cmd & $7,117,227$ & 6,248 & $(431,568)$ \\
\hline courier_medium & $\left(\begin{array}{llll}1 & 2 & 3 & 4\end{array}\right)$ & 2 & 4,245 & 4,245 & $30-1,800$ & $\mathrm{ac}$ & $\mathrm{n} / \mathrm{o}$ & 0 & 30,436 & $(419,400)$ \\
\hline \multirow[t]{3}{*}{ courier_large } & $\left(\begin{array}{llll}1 & 2 & 4 & 3\end{array}\right)$ & 2 & 13,590 & 13,590 & $15-4,830$ & $\mathrm{ac}$ & $\mathrm{n} / \mathrm{o}$ & 0 & 66,137 & $(1,632,600)$ \\
\hline & $\left(\begin{array}{llll}2 & 4 & 1 & 3\end{array}\right)$ & 2 & 3,628 & 2,464 & 450 & ac & $\mathrm{n} / \mathrm{o}$ & $10,325,844$ & 33,618 & $(1,108,800)$ \\
\hline & & & & & & $\mathrm{ac}$ & $\mathrm{cmd}$ & $4,247,436$ & 33,618 & $(1,108,800)$ \\
\hline
\end{tabular}

the numbers of nonzeros in the sparse LU and real Schur factors of the corresponding partitionings. Before the number of nonzeros in the $n z_{L U}$ column, we indicate whether COLAMD has been used (cmd) or not (n/o). Finally, the number in parentheses in the column $n z_{S c h u r}$ gives the number of nonzeros used by the reciprocals of the diagonals of the $T$ factors of candidate blocks which we store explicitly.

In five of the problems, we employ the original ordering of LLMs. When the original ordering does not yield a favorable partitioning in terms of macrostates having a suitable number of candidate blocks that satisfy Proposition 4.1, or the number and order of blocks, it is possible to consider different orderings of LLMs. This we do in kanban_medium, kanban_large, and courier_large. In all problems with the indicated ordering of LLMs in Table 4, there are some candidate blocks. With the original ordering of LLMs in qh_realcontrol, msmq_medium, msmq_large, courier_medium and with the ordering (1 $\left.\begin{array}{llll}2 & 4 & 3\end{array}\right)$ of LLMs in courier_large, all diagonal blocks at the specified partitioning level are candidates, and they satisfy Proposition 4.1. Hence, in the ac, n/o cases of these five problems, there are no nonzeros in the $n z_{L U}$ column and the amount of storage required by the real Schur factors is quite modest. Note that when $n z_{L U}=0$, the number in parentheses in the $n z_{S c h u r}$ column is equal to $n$, as expected. Furthermore, in the three kanban problems, two of which have a single macrostate, only (about) one-sixth of the diagonal blocks are candidates. This is a relatively small percentage compared with the situation in other problems. Hence, benefits of utilizing candidate blocks in the kanban problems will be relatively low compared with other problems. In courier_large, we also consider the ordering (2 4113 ) of LLMs to show that sometimes at the expense of extra storage one may be better off in terms of solution time. We use the real Schur factorization approach only in those candidate blocks that satisfy Proposition 4.1. Hence, even though there are noncandidate blocks in kanban_medium and kanban_large with the ordering (3 42 1) of LLMs, in kanban_fail with the original ordering of LLMs, and in courier_large with the ordering (2 $\left.4 \begin{array}{lll}2 & 1 & 3\end{array}\right)$ of LLMs, the real Schur factors of candidate blocks in these cases are also sparse and require modest storage.

In three of the problems, namely, qh_realcontrol, msmq_large, and kanban_fail, we consider all combinations of ac|nc with cmd and n/o. This yields three cases 
TABLE 5

qh_realcontrol: $\left(\begin{array}{lll}1 & 2 & 3\end{array}\right), l=1, w=1.0$.

\begin{tabular}{l|l|r|l|r|r}
\hline & Solver & it & res & Setup & Solve \\
\hline \multirow{5}{*}{ nc, n/o } & STR_SOR & 3,720 & $10^{-9}$ & 0 & 403 \\
& STR_RSOR & 3,610 & $10^{-9}$ & 0 & 423 \\
& STR_GMRES(20) & 5,000 & $10^{-2}$ & 0 & 993 \\
& STR_BICGSTAB & 3,827 & $10^{-9}$ & 0 & 564 \\
& STR_TFQMR & 5,000 & $10^{-2}$ & 0 & 671 \\
& PRE_GMRES(20) & 5,000 & $10^{-2}$ & 0 & 1,915 \\
& PRE_BICGSTAB & 5,000 & $10^{-8}$ & 0 & 1,570 \\
nc, cmd & PRE_TFQMR & 5,000 & $10^{-7}$ & 0 & 1,520 \\
& BSOR_GOR & 3,430 & $10^{-9}$ & 4 & 414 \\
& BSOR_BICGSTAB & 5,000 & $10^{-1}$ & 4 & 1,494 \\
ac, n/o & 260 & $10^{-9}$ & 4 & 55 \\
& BSOR_TFQMR & 262 & $10^{-9}$ & 4 & 54 \\
& STR_BSOR & 3,430 & $10^{-9}$ & 8 & 422 \\
& BSOR_GMRES(20) & 5,000 & $10^{-1}$ & 8 & 1,466 \\
& BSOR_BICGSTAB & 293 & $10^{-9}$ & 8 & 59 \\
& BSOR_TFQMR & 262 & $10^{-9}$ & 8 & 53 \\
& STR_BSOR & 3,430 & $10^{-9}$ & 1 & 347 \\
& BSOR_GMRES(20) & 5,000 & $10^{-1}$ & 1 & 1,340 \\
& BSOR_BICGSTAB & 276 & $10^{-9}$ & 1 & 53 \\
& BSOR_TFQMR & 256 & $10^{-8}$ & 1 & $\mathbf{4 8}$ \\
\hline
\end{tabular}

in the first two problems and four cases in the last problem. These three sets of experiments, in which LLMs are not reordered and the corresponding problems are of varying difficulty and size, should put the improvements obtained with the proposed solvers into better perspective.

In this study, we consider BSOR preconditioned versions of the projection methods GMRES, BiCGSTAB, and (transpose free) quasi-minimal residual (TFQMR) [24], which are, named, respectively, BSOR_GMRES, BSOR_BICGSTAB, and BSOR_ TFQMR. We compare all results with those of other HMM solvers available in the APNN-toolbox. In particular, we compare BSOR_GMRES, BSOR_BICGSTAB, and BSOR_TFQMR with STR_SOR, STR_RSOR, STR_GMRES, STR_BICGSTAB, STR_ TFQMR, PRE_GMRES, PRE_BICGSTAB, PRE_TFQMR, and STR_BSOR. The STR_SOR solver implements a BSOR-like method which uses the diagonal blocks at level 0 with relaxation parameter $w$ but does not attempt to solve them. When there is a single macrostate, STR_SOR becomes the point Jacobi overrelaxation (JOR) method. The STR_RSOR solver implements a point SOR method with relaxation parameter $w$ similar to the one discussed in [43]. The STR_GMRES solver implements restarted GMRES with a fixed number of vectors for the Krylov subspace, as discussed in $[41$, p. 198]. We use a Krylov subspace size of 20. The STR_BICGSTAB solver implements BiCGSTAB, as discussed in [3, pp. 27-28]. The STR_TFQMR solver implements TFQMR, as discussed in [24]. The PRE_GMRES, PRE_BICGSTAB, and PRE_TFQMR solvers are, respectively, preconditioned versions of STR_GMRES, STR_BICGSTAB, and STR_TFQMR using the cheap and separable preconditioner mentioned in section 3 [11]. Finally, STR_BSOR is the two-level version of the BSOR solver with relaxation parameter $w$ proposed for HMMs in [12].

All experiments were performed on a $3 \mathrm{GHz}$ Pentium IV processor with 1 GByte main memory under Linux. The large main memory is necessary due to the large number of vectors of length $n$ used in projection methods. All times are reported as seconds of CPU time. In Tables 5 through 13, we report the times spent in the setup 
TABLE 6

msmq_medium: (1 $\left.\begin{array}{lllll}1 & 3 & 4 & 5\end{array}\right), l=2, a c, n / o, w=1.0$.

\begin{tabular}{l|r|l|r|r}
\hline Solver & it & res & Setup & Solve \\
\hline STR_SOR & 360 & $10^{-9}$ & 0 & 19 \\
STR_RSOR & 240 & $10^{-9}$ & 0 & 19 \\
STR_GMRES(20) & 5,000 & $10^{-4}$ & 0 & 712 \\
STR_BICGSTAB & 325 & $10^{-9}$ & 0 & 27 \\
STR_TFQMR & 398 & $10^{-9}$ & 0 & 30 \\
PRE_GMRES(20) & 5,000 & $10^{-4}$ & 0 & 1,202 \\
PRE_BICGSTAB & 222 & $10^{-10}$ & 0 & 37 \\
PRE_TFQMR & 226 & $10^{-10}$ & 0 & 38 \\
STR_BSOR & 120 & $10^{-9}$ & 0 & 10 \\
BSOR_GMRES(20) & 119 & $10^{-10}$ & 0 & 25 \\
BSOR_BICGSTAB & 47 & $10^{-9}$ & 0 & 8 \\
BSOR_TFQMR & 46 & $10^{-10}$ & 0 & $\mathbf{7}$ \\
\hline
\end{tabular}

TABLE 7

msmq_large: $\left(\begin{array}{lllll}1 & 2 & 3 & 4 & 5\end{array}\right), l=2, w=1.0$.

\begin{tabular}{l|l|r|l|r|r}
\hline & Solver & it & res & Setup & Solve \\
\hline & STR_SOR & 360 & $10^{-9}$ & 1 & 522 \\
& STR_RSOR & 190 & $10^{-9}$ & 1 & 238 \\
& STR_GMRES(20) & 2,500 & $10^{-4}$ & 1 & 5,023 \\
STR_BICGSTAB & 401 & $10^{-9}$ & 1 & 624 \\
& STR_TFQMR & 484 & $10^{-10}$ & 1 & 672 \\
& PRE_GMRES(20) & 1,000 & $10^{-5}$ & 1 & 5,069 \\
& PRE_BICGSTAB & 403 & $10^{-10}$ & 1 & 1,868 \\
nc, n/o & PRE_TFQMR & 278 & $10^{-11}$ & 1 & 1,208 \\
& STR_BSOR & 120 & $10^{-9}$ & 32 & 201 \\
& BSOR_GMRES(20) & 52 & $10^{-9}$ & 32 & 296 \\
& BSOR_BICGSTAB & 46 & $10^{-9}$ & 32 & 134 \\
& BSOR_TFQMR & 46 & $10^{-10}$ & 32 & 125 \\
nc, cmd & STR_BSOR & 120 & $10^{-9}$ & 74 & 200 \\
& BSOR_GMRES(20) & 52 & $10^{-9}$ & 74 & 194 \\
& BSOR_BICGSTAB & 46 & $10^{-9}$ & 74 & 133 \\
& BSOR_TFQMR & 46 & $10^{-10}$ & 74 & 125 \\
& STR_BSOR & 120 & $10^{-9}$ & 4 & 157 \\
& BSOR_GMRES(20) & 52 & $10^{-9}$ & 4 & 151 \\
& BSOR_BICGSTAB & 46 & $10^{-9}$ & 4 & 113 \\
& BSOR_TFQMR & 46 & $10^{-10}$ & 4 & $\mathbf{1 0 3}$ \\
\hline
\end{tabular}

TABLE 8

kanban_medium: (3 $\left.4 \begin{array}{lll}4 & 2 & 1\end{array}\right), l=2, w=0.9$.

\begin{tabular}{l|l|r|l|r|r}
\hline & Solver & it & res & Setup & Solve \\
\hline & STR_SOR & 3,200 & $10^{-9}$ & 0 & 932 \\
& STR_RSOR & 1,240 & $10^{-9}$ & 0 & 460 \\
& STR_GMRES(20) & 1,380 & $10^{-9}$ & 0 & 550 \\
& STR_BICGSTAB & 959 & $10^{-9}$ & 0 & 274 \\
& STR_TFQMR & 5,000 & $10^{-8}$ & 0 & 1,435 \\
& PRE_GMRES(20) & 440 & $10^{-9}$ & 0 & 405 \\
& PRE_BICGSTAB & 5,000 & $10^{-7}$ & 0 & 3,963 \\
& PRE_TFQMR & 544 & $10^{-11}$ & 0 & 433 \\
ac, n/o & STR_BSOR & 1,110 & $10^{-9}$ & 6 & 165 \\
& BSOR_GMRES(20) & 178 & $10^{-10}$ & 6 & 78 \\
& BSOR_BICGSTAB & 164 & $10^{-9}$ & 6 & 56 \\
& BSOR_TFQMR & 188 & $10^{-9}$ & 6 & 64 \\
& STR_BSOR & 1,110 & $10^{-9}$ & 9 & 196 \\
& BSOR_GMRES(20) & 178 & $10^{-10}$ & 9 & 82 \\
& BSOR_BICGSTAB & 130 & $10^{-9}$ & 9 & $\mathbf{4 7}$ \\
& BSOR_TFQMR & 188 & $10^{-10}$ & 9 & 69 \\
\hline
\end{tabular}


TABLE 9

kanban_large: ( $\left.\begin{array}{llll}3 & 4 & 2 & 1\end{array}\right), l=2, w=0.9$.

\begin{tabular}{l|l|r|l|r|r}
\hline & Solver & it & res & Setup & Solve \\
\hline & STR_SOR & 4,310 & $10^{-8}$ & 0 & 5,010 \\
& STR_RSOR & 1,810 & $10^{-9}$ & 0 & 2,434 \\
& STR_GMRES(20) & 1,720 & $10^{-9}$ & 0 & 2,438 \\
& STR_BICGSTAB & 1,143 & $10^{-9}$ & 0 & 1,333 \\
& STR_TFQMR & 4,682 & $10^{-5}$ & 0 & 5,002 \\
PRE_GMRES(20) & 640 & $10^{-9}$ & 0 & 2,240 \\
& PRE_BICGSTAB & 1,628 & $10^{-6}$ & 0 & 5,006 \\
ac, n/o & PRE_TFQMR & 698 & $10^{-11}$ & 0 & 2,060 \\
& STR_BSOR & 1,630 & $10^{-9}$ & 35 & 886 \\
& BSOR_GMRES(20) & 220 & $10^{-10}$ & 35 & 386 \\
& BSOR_BICGSTAB & 195 & $10^{-9}$ & 35 & $\mathbf{2 7 2}$ \\
& BSOR_TFQMR & 222 & $10^{-9}$ & 35 & 298 \\
& STR_BSOR & 1,630 & $10^{-9}$ & 52 & 1,152 \\
& BSOR_GMRES(20) & 220 & $10^{-10}$ & 52 & 423 \\
& BSOR_BICGSTAB & 195 & $10^{-9}$ & 52 & 312 \\
& BSOR_TFQMR & 222 & $10^{-9}$ & 52 & 334 \\
\hline
\end{tabular}

TABLE 10

kanban_fail: (1 2234 ), $l=2, w=1.0$.

\begin{tabular}{l|l|r|l|r|r}
\hline & Solver & it & res & Setup & Solve \\
\hline & STR_SOR & 1,170 & $10^{-9}$ & 1 & 1,479 \\
& STR_RSOR & 440 & $10^{-9}$ & 1 & 834 \\
& STR_GMRES(20) & 2,900 & $10^{-5}$ & 1 & 5,021 \\
& STR_BICGSTAB & 3,744 & $10^{-8}$ & 1 & 5,004 \\
& STR_TFQMR & 3,900 & $10^{-5}$ & 1 & 5,004 \\
& PRE_GMRES(20) & 1,360 & $10^{-5}$ & 1 & 5,045 \\
nc, n/o & PRE_BICGSTAB & 1,512 & $10^{-4}$ & 1 & 5,006 \\
& PRE_TFQMR & 418 & $10^{-12}$ & 1 & 1,351 \\
& STR_BSOR & 440 & $10^{-9}$ & 6 & 1,738 \\
& BSOR_GMRES(20) & 960 & $10^{-7}$ & 6 & 5,023 \\
& BSOR_BICGSTAB & 480 & $10^{-11}$ & 6 & 2,224 \\
nc, cmd & BSOR_TFQMR & 120 & $10^{-10}$ & 6 & $\mathbf{5 4 5}$ \\
& STR_BSOR & 440 & $10^{-9}$ & 14 & 1,757 \\
& BSOR_GMRES(20) & 960 & $10^{-7}$ & 14 & 5,023 \\
& BSOR_BICGSTAB & 394 & $10^{-11}$ & 14 & 1,819 \\
& BSOR_TFQMR & 120 & $10^{-10}$ & 14 & 551 \\
& STR_BSOR & 440 & $10^{-9}$ & 5 & 1,695 \\
& BSOR_GMRES(20) & 980 & $10^{-7}$ & 5 & 5,072 \\
& BSOR_BICGSTAB & 294 & $10^{-11}$ & 5 & 1,353 \\
& BSOR_TFQMR & 120 & $10^{-10}$ & 5 & $\mathbf{5 4 6}$ \\
& STR_BSOR & 440 & $10^{-9}$ & 12 & 1,722 \\
& BSOR_GMRES(20) & 960 & $10^{-7}$ & 12 & 5,051 \\
& BSOR_BICGSTAB & 328 & $10^{-10}$ & 12 & 1,517 \\
& BSOR_TFQMR & 120 & $10^{-10}$ & 12 & 551 \\
\hline \multicolumn{4}{|l}{}
\end{tabular}

and iterative parts of the solvers, respectively, under the columns Setup and Solve, and we indicate the fastest solvers in bold. In order to improve the confidence in the results, each experiment is run three times and the average of the timings is reported. The it column indicates the number of iterations it takes the solvers to stop, and the res column indicates the infinity norm of the residual upon stopping. In the caption, $l$ stands for level in Table 4 and $w$ is the relaxation parameter. We use a stopping tolerance of $10^{-8}$ on the residual norm (or of its approximation). The maximum number of permissible iterations is 5,000, and the maximum permissible CPU time is 
TABLE 11

courier_medium: (1 2234$), l=2, a c, n / o, w=1.0$.

\begin{tabular}{l|r|l|r|r}
\hline Solver & it & res & Setup & Solve \\
\hline STR_SOR & 1,190 & $10^{-9}$ & 0 & 366 \\
STR_RSOR & 360 & $10^{-9}$ & 0 & 108 \\
STR_GMRES(20) & 5,000 & $10^{0}$ & 0 & 1,962 \\
STR_BICGSTAB & 339 & $10^{-9}$ & 0 & 103 \\
STR_TFQMR & 5,000 & $10^{-1}$ & 0 & 1,447 \\
PRE_GMRES(20) & 4,080 & $10^{-1}$ & 0 & 5,013 \\
PRE_BICGSTAB & 203 & $10^{-11}$ & 0 & 233 \\
PRE_TFQMR & 1,034 & $10^{-10}$ & 0 & 1,120 \\
STR_BSOR & 60 & $10^{-10}$ & 1 & 32 \\
BSOR_GMRES(20) & 38 & $10^{-9}$ & 1 & 32 \\
BSOR_BICGSTAB & 37 & $10^{-9}$ & 1 & $\mathbf{2 7}$ \\
BSOR_TFQMR & 40 & $10^{-9}$ & 1 & 30 \\
\hline
\end{tabular}

TABLE 12

courier_large: (1 $\left.2 \begin{array}{lll}1 & 4 & 3\end{array}\right), l=2, a c, n / o, w=1.0$.

\begin{tabular}{l|r|l|r|r}
\hline Solver & it & res & Setup & Solve \\
\hline STR_SOR & 1,420 & $10^{-9}$ & 0 & 1,797 \\
STR_RSOR & 130 & $10^{-9}$ & 0 & $\mathbf{1 5 9}$ \\
STR_GMRES(20) & 3,200 & $10^{-1}$ & 0 & 5,018 \\
STR_BICGSTAB & 379 & $10^{-9}$ & 0 & 478 \\
STR_TFQMR & 4,090 & $10^{-2}$ & 0 & 5,003 \\
PRE_GMRES(20) & 1,240 & $10^{-2}$ & 0 & 5,050 \\
PRE_BICGSTAB & 254 & $10^{-10}$ & 0 & 951 \\
PRE_TFQMR & 248 & $10^{-11}$ & 0 & 903 \\
STR_BSOR & 60 & $10^{-10}$ & 3 & 253 \\
BSOR_GMRES(20) & 41 & $10^{-9}$ & 3 & 226 \\
BSOR_BICGSTAB & 43 & $10^{-9}$ & 3 & 219 \\
BSOR_TFQMR & 42 & $10^{-9}$ & 3 & 204 \\
\hline
\end{tabular}

TABLE 13

courier_large: (2 $\left.4 \begin{array}{lll}2 & 1\end{array}\right), l=2, w=1.0$.

\begin{tabular}{l|l|r|l|r|r}
\hline & Solver & it & res & Setup & Solve \\
\hline & STR_SOR & 1,420 & $10^{-9}$ & 0 & 1,698 \\
STR_RSOR & 130 & $10^{-9}$ & 0 & $\mathbf{1 4 6}$ \\
& STR_GMRES(20) & 3,320 & $10^{-1}$ & 0 & 5,023 \\
STR_BICGSTAB & 379 & $10^{-9}$ & 0 & 457 \\
STR_TFQMR & 4,358 & $10^{-2}$ & 0 & 5,003 \\
ac, n/o & 1,240 & $10^{-2}$ & 0 & 5,022 \\
& PRE_GMRES(20) & 257 & $10^{-10}$ & 0 & 971 \\
& PRE_BICGSTAB & 246 & $10^{-11}$ & 0 & 900 \\
PRE_TFQMR & 110 & $10^{-9}$ & 4 & 171 \\
STR_BSOR & 77 & $10^{-9}$ & 4 & 213 \\
& BSOR_GMRES(20) & 75 & $10^{-10}$ & 4 & 178 \\
& BSOR_BICGSTAB & 68 & $10^{-9}$ & 4 & 157 \\
& BSOR_TFQMR & 110 & $10^{-9}$ & 7 & 172 \\
& STR_BSOR & 77 & $10^{-9}$ & 7 & 205 \\
& BSOR_GMRES(20) & 74 & $10^{-10}$ & 7 & 170 \\
& BSOR_BICGSTAB & 68 & $10^{-9}$ & 7 & 156 \\
& BSOR_TFQMR & & & &
\end{tabular}

5,000 seconds. In solvers involving BiCGSTAB and TFQMR, each pass through the body of the code counts as two iterations rather than one. We choose to normalize the solution vector and compute the residual every 10 iterations in the solvers STR_SOR, STR_RSOR, and STR_BSOR. Although the BSOR preconditioner in the toolbox has 
the flexibility to sparse LU factorize the diagonal blocks at level 0 corresponding to macrostates that have a small number of microstates, or to use different partitioning levels at different macrostates, these features are turned off. In that sense, the results provided in this section may not be the best that can be obtained with BSOR and BSOR preconditioned projection methods.

For the problems in which convergence is observed due to the stopping tolerance of $10^{-8}$ but the norm of the residual is found to be larger than $10^{-8}$, we continued the iterative process by decreasing the stopping tolerance one order of magnitude at a time until we encountered a residual norm less than $10^{-8}$. Such a situation is witnessed among BSOR preconditioned projection methods since we work with unnormalized solution vectors and the underlying CTMCs are not scaled. Recall that the system we solve is singular and that a nonscaled coefficient matrix with considerably large entries may result in the residual norm being larger than what the (unnormalized) solution vector actually implies (see [18, p. 1697]), especially when convergence takes place rapidly. In only one of the problems are we not able to reduce the residual norm below $10^{-8}$ by iterating in this manner, and that happens to be with the BSOR_TFQMR solver using ac, $\mathrm{n} / \mathrm{o}$ factorization in qh_realcontrol where the residual norm is computed to be in the order of $10^{-8}$.

It is not easy to make a general statement about the value of the optimal relaxation parameter in SOR-type methods for MCs. A study of this kind was done in [18] using SOR and BSOR on sparse MC problems. In many test problems considered therein, the optimal value of the relaxation parameter, $w$, turned out to be 1.0 (see [18, pp. 1700-1701]). In our experiments in this paper, $w$ is set to 1.0, except for the kanban_medium and kanban_large problems, in which it is set to 0.9 due to the fact that their reordered smaller version in Example 2 does not converge using STR_RSOR and STR_BSOR with $w=1.0$ for the chosen partitioning. Since there are already too many parameters to set in the BSOR preconditioner for HMMs, we have concentrated on a few of them that would help us evaluate the merits of using the proposed ideas in BSOR preconditioned solvers.

In the next section we summarize the results of our numerical experiments.

7. Summary of results. The setup time of the BSOR preconditioner turns out to be a relatively small fraction of the total solution time with BSOR preconditioned solvers when all diagonal blocks are candidates and they are real Schur factorized (see Tables 5, 6, 7, 11, and 12). This is due to the fact that in all such cases in this paper, the real Schur factors of candidate blocks can be constructed from component matrices and their real Schur factors. In the ac, n/o cases of qh_realcontrol, msmq_medium, msmq_large, courier_medium, and courier_large with the ordering (1 2443$)$ which fall under this category, memory requirements for the factors of the diagonal blocks are relatively low (see Table 4 ). The ac, n/o case of msmq_large yields more than a fifteenfold decrease in storage allocated to the factors of the diagonal blocks compared with that of its nc, n/o case. Note that the ratio of the setup times of the nc, cmd and ac, $\mathrm{n} / \mathrm{o}$ cases in the msmq_large problem is also relatively large and equal to 18.5 (see Table 7). Hence, we can say that there are significant savings in storage and setup time with the BSOR preconditioner when all diagonal blocks are candidates whose real Schur factors can be constructed from component matrices and their real Schur factors. We remark that this can be done in five out of the eight problems in Table 3.

With regards to storage savings due to COLAMD in the nc cases, there is a $33 \%$ reduction in qh_realcontrol, a 35\% reduction in msmq_large, and a $36 \%$ reduction in kanban_fail. With regards to storage savings due to COLAMD in the ac cases, 
there is a $35 \%$ reduction in kanban_fail and a $53 \%$ reduction in courier_large with the ordering (2 $\left.4 \begin{array}{lll}2 & 3\end{array}\right)$ of LLMs. These also translate to significant storage savings. Note that, as we remarked in section 4.4, fill-in increases in the kanban_medium and kanban_large problems if COLAMD is used. On the other hand, the setup time of the BSOR preconditioner increases when COLAMD is used (see Tables 5, 7, 8, 9, 10, and 13). However, this increase is never more than twice the setup time of the corresponding $\mathrm{n} / \mathrm{o}$ case. The difference between the setup times of kanban_large and kanban_fail is due to the difference between the order of diagonal blocks that get sparse LU factorized in each case.

In all problems there are at least two BSOR preconditioned projection methods among the fastest five solvers (see Tables 5-13). If we exclude the courier_large problem, the winner is BSOR_TFQMR four times and BSOR_BICGSTAB three times. The BSOR_GMRES(20) solver appears only a total of five times among the fastest five solvers in Tables 5-13. Results of this kind have also been observed in [18, 37]. Especially in such large problems as those considered in this paper, the Krylov subspace size seems to be the bottleneck in obtaining a competitive GMRES solver. To the contrary of GMRES, there is only a fixed, small number of vectors that need to be employed with BiCGSTAB and TFQMR. Note that in msmq_large, BSOR_GMRES(20) with the nc, $\mathrm{n} / \mathrm{o}$ factorization of diagonal blocks is using virtual memory due to the value of $n z_{L U}$ and the space that must be allocated to more than 20 vectors, each having a length of about 3 million. This is why its solution time is much longer than those of the nc, cmd and ac, n/o cases. We also remark that any discrepancy in the number of iterations of BSOR preconditioned solvers for a problem across its ac|nc cases with cmd and n/o is due to floating-point arithmetic.

STR_RSOR is the winner in the courier_large problem with the ordering (1 2 4 3) of LLMs; BSOR_TFQMR and BSOR_BICGSTAB follow as second and third, respectively. Observe that in this case the orders of diagonal blocks in the BSOR preconditioner are relatively nonuniform compared with those of other cases. By reordering the LLMs as $\left(\begin{array}{lllll}2 & 4 & 1 & 3\end{array}\right)$ we obtain diagonal blocks of order 450 . Even though not all diagonal blocks are candidates in this alternative ordering, by investing more storage in the factorization of diagonal blocks, one is able to obtain a stronger BSOR preconditioner, which makes BSOR_TFQMR a closer second. Nevertheless, the courier_large problem is a good example, showing that sometimes STR_RSOR will yield the fastest solver. Results of this kind have also been observed in [18].

STR_BSOR and STR_RSOR are among the fastest five solvers five and four times, respectively. When there is sufficient decrease in the number of iterations to convergence with STR_BSOR, STR_BSOR is faster than STR_RSOR. We remark that the right-hand-side update that takes place due to the (block) strictly uppertriangular part at each iteration in STR_RSOR and STR_BSOR is detrimental to the efficient vector-Kronecker product multiplication algorithm. In kanban_medium and kanban_large, which are two problems with one macrostate, nonzeros of the underlying CTMCs are constrained mostly within the diagonal blocks of the chosen partitionings. Therefore, even if the decrease in the number of iterations in these two problems with STR_BSOR is marginal, STR_BSOR performs much better than STR_RSOR timewise. This cannot be said for the kanban_fail and courier_large problems, in which the two-level version of STR_BSOR is at a disadvantage. Nevertheless, it is possible to consider the three-level version of the STR_BSOR solver in $[12]$ as well.

There is significant decrease in the number of iterations to convergence when 
BSOR is used as a preconditioner with projection methods. In fact, for those cases in which both unpreconditioned and BSOR preconditioned projection methods converge within the experimental framework, the number of iterations with the BSOR preconditioned solver is at most one-fifth that of the unpreconditioned solver. Furthermore, there are a few cases in which the ratio is about one-tenth. Although the cheap and separable preconditioner performs well on some problems, it is clearly inferior to the BSOR preconditioner. In conclusion, BSOR preconditioned BiCGSTAB and TFQMR solvers can be recommended for HMMs.

8. Conclusion. CTMCs in the form of sums of Kronecker products have considerable structure that may be exploited in devising effective preconditioners for projection methods. A two-level BSOR preconditioner that exploits this structure is presented for HMMs. The idea of using one real Schur factorization per macrostate for the diagonal blocks of the BSOR preconditioner that differ from each other by a multiple of the identity (that is, candidate blocks) and using COLAMD ordering in the remaining diagonal blocks tends to reduce storage taken by the BSOR preconditioner. When there is a relatively large number of candidate blocks and they all meet certain conditions (as in Proposition 4.1), the setup time of the BSOR preconditioner is expected to be relatively small compared with the total solution time of the BSOR preconditioned solver. To improve the situation for the BSOR preconditioner, one may consider different orderings of LLMs. Numerical experiments on a representative set of problems demonstrate that BSOR preconditioned BiCGSTAB and TFQMR using these ideas are potentially effective solvers for Kronecker structured Markovian representations.

Acknowledgment. We thank the anonymous referees for their constructive reports, which led to an improved manuscript.

\section{REFERENCES}

[1] M. Ajmone-Marsan, S. Donatelli, And F. Neri, GSPN models of Markovian multiserver multiqueue systems, Performance Evaluation, 11 (1990), pp. 227-240.

[2] APNN-ToolBox, http://www4.cs.uni-dortmund.de/APNN-TOOLBOX/.

[3] R. Barrett, M. W. Berry, T. F. Chan, J. Demmel, J. Donato, J. Dongarra, V. Eijkhout, R. Pozo, C. Romine, And H. van Der Vorst, Templates for the Solution of Linear Systems: Building Blocks for Iterative Methods, SIAM, Philadelphia, 1994.

[4] F. Bause, P. Buchholz, and P. Kemper, A toolbox for functional and quantitative analysis of DEDS, in Quantitative Evaluation of Computing and Communication Systems, $\mathrm{R}$. Puigjaner, N. N. Savino, and B. Serra, eds., Lecture Notes in Comput. Sci. 1469, SpringerVerlag, New York, 1998, pp. 356-359.

[5] M. BenzI, Preconditioning techniques for large linear systems: A survey, J. Comput. Phys., 182 (2002), pp. 418-477.

[6] M. Benzi And M. Tuma, A parallel solver for large-scale Markov chains, Appl. Numer. Math., 41 (2002), pp. 135-153.

[7] A. Berman and R. J. Plemmons, Nonnegative Matrices in the Mathematical Sciences, SIAM, Philadelphia, 1994.

[8] P. Buchholz, A class of hierarchical queueing networks and their analysis, Queueing Systems Theory Appl., 15 (1994), pp. 59-80.

[9] P. Buchiolz, Hierarchical structuring of superposed GSPNs, IEEE Trans. Software Engrg., 25 (1999), pp. 166-181.

[10] P. BuchHolz, Structured analysis approaches for large Markov chains, Appl. Numer. Math., 31 (1999), pp. 375-404.

[11] P. Buchuolz, Projection methods for the analysis of stochastic automata networks, in Numerical Solution of Markov Chains, B. Plateau, W. J. Stewart, and M. Silva, eds., Prensas Universitarias de Zaragoza, Zaragoza, Spain, 1999, pp. 149-168. 
[12] P. BuchHolz And T. DAYAR, Block SOR for Kronecker structured representations, Linear Algebra Appl., 386 (2004), pp. 83-109.

[13] P. Buchiolz And P. Kemper, On generating a hierarchy for GSPN analysis, Performance Evaluation Rev., 26 (1998), pp. 5-14.

[14] P. Buchholz, G. Ciardo, S. Donatelli, and P. Kemper, Complexity of memory-efficient Kronecker operations with applications to the solution of Markov models, INFORMS J. Comput., 12 (2000), pp. 203-222.

[15] J. Campos, S. Donatelli, AND M. Silva, Structured solution of asynchronously communicating stochastic models, IEEE Trans. Software Engrg., 25 (1999), pp. 147-165.

[16] R. H. Chan AND W. K. Ching, Circulant preconditioners for stochastic automata networks, Numer. Math., 87 (2000), pp. 35-57.

[17] COLAMD, Column Approximate Minimum Degree Ordering, http://www.cise.ufl.edu/ research/sparse/colamd/.

[18] T. Dayar And W. J. Stewart, Comparison of partitioning techniques for two-level iterative solvers on large, sparse Markov chains, SIAM J. Sci. Comput., 21 (2000), pp. 1691-1705.

[19] J. W. Demmel, Applied Numerical Linear Algebra, SIAM, Philadelphia, 1997.

[20] S. Donatelli, Superposed stochastic automata: A class of stochastic Petri nets with parallel solution and distributed state space, Performance Evaluation, 18 (1993), pp. 21-26.

[21] P. Fernandes, B. Plateau, and W. J. Stewart, Efficient descriptor-vector multiplications in stochastic automata networks, J. ACM, 45 (1998), pp. 381-414.

[22] P. Fernandes, B. Plateau, and W. J. Stewart, Optimizing tensor product computations in stochastic automata networks, RAIRO Rech. Opér., 32 (1998), pp. 325-351.

[23] R. W. Freund And M. HochBruck, On the use of two QMR algorithms for solving singular systems and applications in Markov chain modelling, Numer. Linear Algebra Appl., 1 (1994), pp. 403-420.

[24] R. W. Freund, A transpose-free quasi-minimal residual method for non-Hermitian linear systems, SIAM J. Sci. Comput., 14 (1993), pp. 470-482.

[25] A. Greenbaum, Iterative Methods for Solving Linear Systems, SIAM, Philadelphia, 1997.

[26] P. KemPeR, Numerical analysis of superposed GSPNs, IEEE Trans. Software Engrg., 22 (1996), pp. $615-628$.

[27] A. N. Langville Preconditioning for Stochastic Automata Networks, Ph.D. Thesis, Operations Research, North Carolina State University, Raleigh, 2002; available online from http://www.lib.ncsu.edu/etd/public/etd-232913131021900/etd-title.html.

[28] A. N. LAngVille And W. J. Stewart, A Kronecker product approximate preconditioner for SANs, Numer. Linear Algebra Appl., 11 (2004), pp. 723-752.

[29] V. Migallón, J. Penadés, And D. B. Szyld, Block two-stage methods for singular systems and Markov chains, Numer. Linear Algebra Appl., 3 (1996), pp. 413-426.

[30] D. Mitra and I. Mitrani, Analysis of a Kanban discipline for cell coordination in production lines II: Stochastic demands, Oper. Res., 39 (1991), pp. 807-823.

[31] Netlib, A Collection of Mathematical Software, Papers, and Databases, http://www.netlib.org.

[32] B. Philippe, Y. SaAd, And W. J. Stewart, Numerical methods in Markov chain modelling, Oper. Res., 40 (1992), pp. 1156-1179.

[33] B. PlateAu, On the stochastic structure of parallelism and synchronization models for distributed algorithms, in Proceedings of the ACM SIGMETRICS Conference on Measurement and Modelling of Computer Systems, Austin, TX, 1985, pp. 147-154.

[34] B. Plateau And K. Atif, Stochastic automata network for modeling parallel systems, IEEE Trans. Software Engrg., 17 (1991), pp. 1093-1108.

[35] B. Plateau and J.-M. Fourneau, A methodology for solving Markov models of parallel systems, J. Parallel Distrib. Comput., 12 (1991), pp. 370-387.

[36] Y. SAAD, Projection methods for the numerical solution of Markov chain models, in Numerical Solution of Markov Chains, W. J. Stewart, ed., Marcel Dekker, New York, 1991, pp. 455471.

[37] Y. SAAD, Preconditioned Krylov subspace methods for the numerical solution of Markov chains, in Computations with Markov Chains: Proceedings of the Second International Workshop on the Numerical Solution of Markov Chains, W. J. Stewart, ed., Kluwer Academic, Boston, MA, 1995, pp. 49-64.

[38] Y. SAAD, Iterative Methods for Sparse Linear Systems, 2nd ed., SIAM, Philadelphia, 2003.

[39] Y. SAAd And M. H. Schultz, GMRES: A generalized minimal residual algorithm for solving nonsymmetric linear systems, SIAM J. Sci. Statist. Comput., 7 (1986), pp. 856-869.

[40] G. W. Stewart, Matrix Algorithms, Volume II: Eigensystems, SIAM, Philadelphia, 2001.

[41] W. J. Stewart, Introduction to the Numerical Solution of Markov Chains, Princeton University Press, Princeton, NJ, 1994. 
[42] W. J. Stewart, K. Atif, and B. Plateau, The numerical solution of stochastic automata networks, European J. Oper. Res., 86 (1995), pp. 503-525.

[43] E. UYSAL AND T. DAYAR, Iterative methods based on splittings for stochastic automata networks, European J. Oper. Res., 110 (1998), pp. 166-186.

[44] H. A. VAN DER Vorst, Bi-CGSTAB: A fast and smoothly converging variant of Bi-CG for the solution of nonsymmetric linear systems, SIAM J. Sci. Statist. Comput., 13 (1992), pp. 631-644.

[45] C. F. VAn LoAn, The ubiquitous Kronecker product, J. Comput. Appl. Math., 123 (2000), pp. $85-100$.

[46] C. M. Woodside And Y. Li, Performance Petri net analysis of communications protocol software by delay equivalent aggregation, in Proceedings of the 4th International Workshop on Petri Nets and Performance Models, IEEE Computer Society Press, Los Alamitos, CA, 1991, pp. 64-73. 